\title{
GENERATION OF RAYLEIGH WAVES
} BY UNDERGROUND NUCLEAR EXPLOSIONS: AN EXAMINATION OF THE EFFECT OF SPALL IMPACT AND SITE CONFIGURATION

\author{
J. A. Viecelli \\ (Ph,D. Thesis)
}

July 3,1973

Prepared for U.S. Atomic Energy Commission under contract No. W-7405-Eng-48

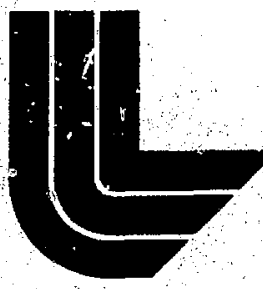

\section{LAWRENCE} LIVERMORE LABORATORY

University of Californiay/Livermore

\section{MASTER}


(1) $13-4500, \mathrm{CC}-3.4$

Ehysirs-General

\title{
니 \\ LAWRENCE LNEFMORE LAEORATOFY
}

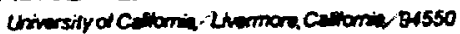

UCRL-51417

\section{GENERATION OF RAYLEIGH WAVES \\ BY UNDERGROUND NUCLEAR EXPLOSIONS: \\ AN EXAMINATION OF THE EFFECT OF SPALL IMPACT AND SITE CONFIGURATION}

\author{
J. A. Viecelli \\ (Ph.D. Thesis)
}

MS. date: july 3,1973

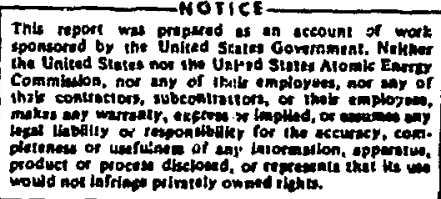




\section{Contents}

Abstract

1. Introduction and Summary of Work . . . . . . . . . . . . . . . 3

I1. Listimation of spall Parameters . . . . . . . . . . . . . 10

A. Jiscustion . . . . . . . . . . . . . . . 10

B. Spall Dimensions and Volocily . . . . . . . . . . 11

C. Mass and Momentum of Spall . . . . . . . . . . . . 14

ilj. Numericisl Mathods . . . . . . . , . . . . . 17

A. Discussion . . . . . . . . . . . . . . 17

B. Analysis of Artificial Viscosity . . . . . . . . . . . . . 18

C. Computation of Surfare Waves . . . . . . . . . . . . . 26

JV. Calculation of Spall . . . . . . . . . . . . 37

A. Discussion . . . . . . . . . . . . . . . . . 37

B. Mathematical iviodels of the Mechanical Properites of Rocks . $\quad 39$

C. Comparison of Calculations with Bxperiment . . . . . . . . 45

y. Rayleigh-Wave Generation by Spall Inpact . . . . . . . . . 49

A. Predictions of Idealized Model Compared with Experiment . . $\quad$ 49

B. Predictions of Computer Models . . . . . . . . . 5l

C. Conclusions . . . . . . . . . . . . . . 55

VI. Site Geometry Effects . . . . . . . . . . . . . . . 58

A. Introduction . . . . . . . . . . . . . . . 58

B. Calculations . . . . . . . . . . . . . . . . . 58

C. Results and Conclusions . . . . . . . . . . . . . . 61

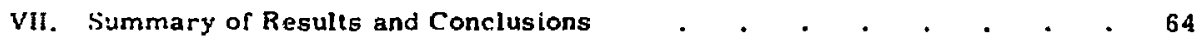

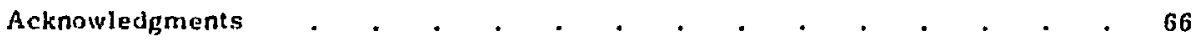

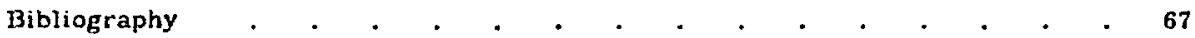




\title{
GENERATION OF RAYLEIGH WAVES BY UNDERGROUND NUCLEAR EXPLOSIONS: AN EXAMINATION OF THE EFFECT OF SPALL IMPACT AND SITE CONFIGURATION
}

\begin{abstract}
The mechanism of Rayleigh-wave generation by underground nuclear explosions has been examined by experimental and computational methods. The subject is of interest because the principal teleseismir method of discriminating between nuclear tests and earthquakes is based on a comparison of Rayleigh-wave amplitudes.

Underground nuclear explosions, even though thoroughly contained, invariably produce spallation. This mass of earth and rack is kicked up by the initial shock wave and falis back within a second or two. The hypothesis that the impact of this material is a source of the surface waves observed from explosions was investigated. The hypothesis was tested by estimating the spall impulse from acceleromeier data, computing the surface wave amplitudes consistent with the impulse, and then comparing the results with observed amplitudes. It was also checked by performing computer explosion-simulation experiments, with and without spallation, and comparing the amplitudes of the resulting surfate waves. The comparison of amplitudes derived from spall momentum with the observed Rayleigh amplitudes at ranges or 50 to $500 \mathrm{~km}$ shored that the spall has sufficient impulse to account for these waves.

The romputer simulation cxperiments also showed that the Rayleigh amplitudes corresponding to realistic inelastic spallation have several times the amplitude of Rayleigh waves computed from purely elastic behavior. The surface wave for the spallation case is also delayed relative to the wave irorn the elastic source.

Computer madels were also used to estimate the Rayleigh - wave generating efficiency of different configurations of dilatational sources beneath different topographies. Concentrated sources and vertically and horizontally distributed sources placed beneath mountains, canyons, and plains were considered. Signals from a fixed energy release were compared in the time dimain. The strongest signal had four times the amplitude of the weakest. The strongest signal was produced by a horiesitally distributed source placed beneath a mountain, and the weakest signal was produced by a concentrated source placed beneath a nat plain. The horizontal dimension of the source had the biggest effect on the Rayleigh amplitude.

The use of Larmangian finite difference methods in the rumerical studies required an analysis of the effect of the linear $Q$ on the decay of a spherical shock
\end{abstract}


wave in a solid and on the decay of a propagating surface wave. This analysis was accomplished by solution of the partial differential eguations approximated by the difference equations. The computer solutions approach an asymptotic form determined by the linear $Q$. with a decay rate determined by the linear Q. It was determined that to obtain accurate consistent numerical auswerg it would be necessary to replace the "artificlal viscosity" coefficients by input physical coefficients. This was done.

Finally, detalled numerical calculations of the Boxcar and Gasbuggy nuclear explosions were performed. These calculations covered the region between the detoration point and the surface, and extended out to a radial distance of slightly greater than $1 \mathrm{~km}$. The constitutive relations describing the material properties were based on laboratory measurements of the mechanical properties of the rocks obtained from the drill holes at the re. spective sites. The calculations followed the expansion of the vaporized rock at the detonation point, the propagation of the shock to the surface, and the subsequent reflection and spall. Spall impulse and surface displacement profiles obtained from the numerical solutions were in reasonable agreement with values inferred from accelerometers at the respective test sites. 


\section{Introduction and Summary of Work}

Geophysicists have long been interested in. the study of explosion-generated seismic waves for scientifit and industrial purposes. With the beginring of underground nuclear weapons testing, renewed interest in the subject has developed because of the political and military importance of possible claridestine testing. This new field of "nuclear-explosion seismology" is basically concerned with 1) detecting and locating the source of spismic signals and 2) discriminating between explosion-generated signals and signals produced by earthquakes. Thus its objectives differ from the older siudies, whose primary emphasis was on deducing information about the structure of the earth.

If an underground explosion is buried deeply enough no earth wili be thrown up and no crater will be formed at the tim 3 of detonation. At some later time, varying between a few seconds and several months, the ground surface above the shot point may subside. These explosions are called "contained," since there is little or no release of radioactivity into the atmosphere. Thy first contained underground nuclear explosion was the $1.7-k t$ event, Rainier, which was fired in tuff (a $k$ ind of compacted partially fused volcanic ash) at the Nevada Test Site in 1957. Seismii signals from this explosion were detected throughout most of the western United States and as far away as Alaska.

An increase in the explosive energy will produce a stronger seismic signal, which can be detected at greater distances from the explosion. The 5,3-kt contained explosion, Salmon, which was detonated in salt near Hattiesburg, Misaissippi, in 1964, produced aeismic signals that were detected in Bolivia, Scandinavia, France and Czechoslovakia. The much larger, 80-kt, contained explosion, Longshot, was detonated in andesite (a type of volcanic rock) on Amchitka (sland in the Aleutians in 1965 and produced seismic signals that were detected all over the world.

The seismic waves detected are mainly elastic waves of four types. In order of their arrival they are P-waves, S-waves, Love waves, and Rayleigh waves. A P-wave is a wave of compressions and rarifactions, similar to a sound wave. An S-wave is a wave-transmitting shearing motion. Both of thesis waves are called "body waves" because they can travel through the bulk of the earth. The other two types, Rayleigh and I,ove waves, are called "surface waves" because their energy propagates parallel to the surface, decaying exponentially with depth.

Rayleigh waves require only a free surface to propapate, whereas Love waves require, in addition, a layered geological structure. A Rayleigh wave causes a point on the surface to trace out an ellipse in a vertical plane passing through the source. A I ove wave causes the point to move back and forth horizontally in a plane perpandicular to a line through the source.

In addition to the four basic types there are other kinds of waves, such as head waves, which are body waves but which transmit ccmpressions, rarefactions. and shearing motions simultaneously. 
The other types of waves, however, are not of importance in long-range detection and discrimination.

The velocity of the waves depends on the type of wave and the material through which it travels. Generally, in uniform materials, S-waves and Love waves travel at a little over one-half the P-wave velocity, and Rayleigh waves travel at about one-half the P-wave visocity. In unconfined soft rocks, such as tuffs, the $P$-wave velocity varies between 2 and $3 \mathrm{~km} / \mathrm{sec}$, whereas in unconfined harder rock, such as granites, the velocity is from 4 to $6 \mathrm{~km} / \mathrm{sec}$. As rocks are com pressed, the bulk and shear moduli increase faster than the density, so that the $P$-wave and shear wave velocities increase with increasing confining pressure. This phenomenori and changes in material composition and phase account for the fact that the $P$-wave velocity measured from travel tines througt. the Earth depends on the depth the wave traverses. From traveltime measurements seismologists have deduced that the $P$-wave velocity reaches at least $6 \mathrm{~km} / \mathrm{sec}$ at a depth of $5 \mathrm{~km}$ beneath continents and then jumps to $8 \mathrm{~km} / \mathrm{sec}$ at the base of the crust, the Mohorovicic discontinuity. The general trend of the P-wave velocity is an increase with depth, finally attaining a value of roughly $14 \mathrm{~km} / \mathrm{sec}$ at the coremantle boundary.

The $P$-waves and $S$-waves follow Snell's Law. Waves initially headed down into the Earth at an angle get refracted back to the surface because of the increase in velocity with depth and the earth's curvature. If $\theta$ is the angle defined by the explosion aite, the center of the Earth, and the receiving atation, then signals received out to about $10^{\circ}$ come from waves propagating througk the crust and upper mantle. Betweon $10^{\circ}$ and $25^{\circ}$, signals tend to be weak and erratic. It is beilieved that this "shadow zone" is caused by a low-velocity layer, a few tens of kilometers thick, lying beneath the Mohorovicic discontinuity. Tha waves that would ordinarily propagate? out to the region between $10^{\circ}$ and $25^{\circ}$ get deflected deeper into the Earth by the low-velocity layer. Beyond $25^{\circ}$ the signals become more reliable. This region is called the teleseismic zone and is the region of must interest in nuclearexplosion seimology. The $P$-wave and $S$-wave energy arriving in this cone is the fraction of the explosion energy radiated through a cone, extending down below the explosion site, with a half angle of only about $15^{\circ}$. The rest of the energy coming out at wider angles is all refracted back to the surface at ranges corresponding to $\theta$ less than $25^{\circ}$.

The Rayleigh and Love waves, of course, stay confined to the surface, but because the Earth is not uniform their velocity depends on wave length. The longer-wave-length waves sample a thicker section of the crust where the velocities are higher and so travel at a higher velocity than the short-period waves, which sample only the lowestvelocity layers of the Earth at the surface. The velocity varies between about $1.0 \mathrm{~km} / \mathrm{sec}$ for the shortest-period waves to about $4.5 \mathrm{~km} / \mathrm{sec}$ for the longest period.

Body waves and surface waves re also distinguishable by their spectrum. The Earth acts as a low-pas: filter, so that for both types of waves the maximum. in the spectrum recorded at teleseismic 
range occurs for relatively long wave lengths. For body vaves the peak in the spectrum occure at about $1.0 \mathrm{sec}$, while for surface waves the peak is around 20.0 sec. Because of this, seismological stations usually have two sets of instruments: short-period seismographs tuned to have meximum sensitivity in the 1 - to 2-sec period range for receiving $P$-waves arid S-waves, and longer-period instrurrents normally tuned to maximum sensitivity in the $10-$ to $30-\mathrm{sec}$ range for receiving the Rayleigh and Love waves. There is a limit to the amplification that can be used, because there is always a certain amount of background noise present; the Earth is never absolutely quiet.

The dependence of the signal amplitude upon frequency, wave type, and angle between the source and detector, $\theta$, has led seismologists to devise several complicated magnitude scales for measuring the strength of an earthquake or explosion. The scales relat 2 g to the teleseismic zone are $m$, the body wave magnitude, and $M$, the surface-wave magnitude. The purpose of these scales is to assign a single number to an explosion or earthquake that will be independent of $\theta$ and be representative of the strerigth of the event. The relationship between the displacement amplitude measured by the seismograph and the magnitude has the form

$$
\left[\begin{array}{l}
m \\
M
\end{array}\right]=\log _{10}(A / T)+\left[\begin{array}{l}
f_{1}(\theta) \\
f_{2}(\theta)
\end{array}\right]
$$

where $f_{1}$ and $f_{2}$ are functions that compensate for geometrical spreading and absorption, $\mathbf{A}$ is the maximum peak-to- peak displacement, and $T$ is the period associated with, the maximum amplitude.

The number of earthquakes occurring worldwide per year is a very strong function of body wave magnitude. Only 50 to 100 earthquakes with body magnitude gr sater than 6 occur in any given year. but there are as many as 10,000 carthquakes per ycar with body-wave inagnitudes greater than 4.0. Magnitude 4.0 is approximately th: teleseismic detection threshold. Nuclear explusions as small as $1 \mathrm{kt}$, when fired in a hard material such as granite, produce body-wave magnitudes as large as 4.0. Even in a soft medium such as partly saturated alluvium, a yield as small as $20 \mathrm{kt}$ can prrdice brody wave magnitudes as large a 4.0. Because of this it is necessary, have some means of differentiating between signals from earthquakes and si: nals from explosions.

The search for discriminent crite ta has mainly been an empirical one bs ed on comparisions of earthquake and explotion recordings. Evernden ( 969) gives a comprehensive discussion of the various criteria that have been proposed. The main observed teleseismic differences between explosions and earthquakes are that earthquakes generally produce larger-amplitude Rayleigh waves, Love waves, and $S$-waves. Tha most thoroughly examined criterion is the $m: M$ disciminant (Brune et al.. (963); Press et al., 1963; SIPRI, 1969; Liebermann and Pomeroy. 1969). This is based on the observation that, for an earthquake and an explosion producing identical body-wave magnitudes, the surface-wave magnitude for the earthquake is about one unit larger than the surface-wave inagnitude for the explosion. 
In other words, the earthquake produces 10 times larger surface-wave amplitudes that: ie explosion, for the same body-wave amplitude. Figure 1 (taken from SIPRI, 1968) shows a scatter diagram for a 'arge number of earthquakes and explosions. The coordinates of the diagram are the surface-wave matnitude (horizontal axis) and the body-wave magnitude (vertical axis). Figure 1 shows a clear separation between the cluster of earthquake data points and the explosion population.

This discriminant has been developed from an empirical approach, The reason why earthquakes have highermagnitude surface waves than explosions of the same body-wave magnitude has not been explained theoretically. The success of the $\mathrm{m}: \mathrm{M}$ discriminant is the reason why there is considerable practical interest in gaining an understanding of how explosions generate Rayleigh waves. Such an understanding might inc'icate how to increase the Rayleigh - wave output and so p: ovide a means of evading the surface

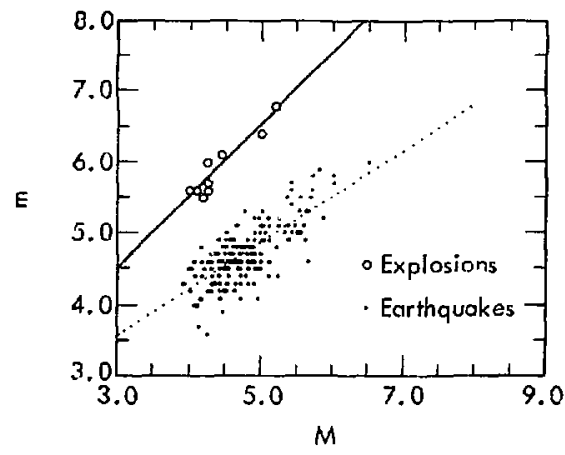

Fig. 1. The separation of explosions and earthquake $;$ by the $\mathrm{m}: \mathrm{M}$ criterion. The body wave magnitude is $m$ and the surface wave mannitude is $M$. wave magnitude-body wave magnitude discriminant.

The first person to attemint a theoretical treatment of Rayleigh waves from ar. explosion was Lamb (1904). In orjer to make the problem mathematicaliy tractable he assumed 1) that ihe eartil can be represented by a uniform elastic half space, 2) that the earth responds linearly to all applied forces, and 3 ) that the explosion itself can be represented by a point source of dilatation with an impulsive timedependence. His solution was in the form of integrals that he could evaluate only asymptotically, far from the explosion center.

Nakano (1925) made the next important theoretical contribution. He made the same assumptions as Lamb and, in addition, restricted his model to two spatial dimensions so that the source in his model was a buried infinite-length line parallel to the surface. He had difficulties similar to Lamb's in evaluating his solution, but he did succeed $i$ i showing that the Rayleigh wave has no clear definition at the scurce.

Garvin (1956) finally succeeded in getting an exact solution for a buried line source with an approximately step-like time dependence for the dilatation. Because of the time dependence the solution contains a number of singularities and requires much mathematical computation for its evaluation.

Hannon (1972) recently gave a comprehensive discussion of the elastic theory of Rayleigh wave generation based on Garvin's solution method.

During the years 1957 to 1968 an increasing number of tests were conducted underground, and investigators began to 
seek a better approximation for an explosive source. Up until that time no attempts had been made to relate the parameters of the simple sources directly to the explosion. Instead, the source parameters were adjusted to give the closest match between theoretical and experimental seismograms.

Carpenter et al. (1962) and Werth et al. (1962) made the first attempts to remedy this situation by introducing the concept of reduced displacement potential and equivalent elastic source. This model assumes that, in a uniform medium, there always exists a time-dependent point source of displacement potential that, beyond some distance from the explosion defined as the elastic radius, produces exactly the same displacements as the real explosion. Werth and Herbst (1963) used displacement data obtained at ranges of a few hundred meters from nuclear explosions to deduce reduced displacement potentials for explosions in tuff, salt, granite, and alluvium. These results were ignored in Rayleigh wave calculations until Marshall (1970) used them to theoretically calculate $\mathrm{m}: \mathrm{M}$ relationships for explosions. His results are in fairly close agreement with the teleseismic data of Licberman and Pomeroy (1969) and also the data of Thirlaway, Pasechnik, Press, and Whitham appearing in SLPRI (1968).

Marshall's calculations assume that the sphere corresponding to the elastic radius of the explosion I ies well below the surface and that the earth behaves elastically everwhere outside this sphere. In the case of a nuclear explosion it is unlikely that this assumption is ever valid, because of the phenomena of sur- face spallation. Spallation occurs when the compression shock from the explosion strikes the surface and reflects as a tension pulse traveling back into the rock, At some depth the tensile stress in the downward-traveling wave excerds the sum of the rock's tensile strength, the lithostatic stress (the pressure due to gravity analogous to hydrostatic pressure in a liquid), and the compressive stress remaining in the tail end of the upwardtraveling compression wave. At that depth the rock parts, trapping a substantial portion of the shock wave momentum in a layer of rock, which flies up from the surface. W. R. Perret of Sandia Laboratories has made most of the closein (ground zero to several kilometers) measurements of ground motion for the U.S. underground nuclear tests. Much of these data are uspublished; however, Eisler and Chit+ra ( afA): Eisler et al. (1966); and Chilton et al. (1966), of Stanford Researciı Institute, analyzed some of Perret's early data and published both a description of the spall phenomenon and some of the early accelerometer records. The surface-motion data ihow that, over a large area above the explosion, the verticel surface displacements are at least 5 to 10 times greatcr than what would be expected on the basis of linear elastic response with the appropriate reduced displacement potential source. In spite of the fact that Marshall (1970) obtained reasonably good results in predicting the explosion $\mathrm{m}: \mathrm{M}$ relationship on the basis of linear elastic theory and the reduced displacement potential source, it is clear from the slose-in displacement measurements that nonlinear inelastic phenomena, such as spall, must play 
some role in the Rayleigh-wave generating process. Another reason for questioning the adequacy of the point-source reduceddisplacement-potential model is that a nuclear test can invorve multiple explosions with overlapring elastic radii. In these cases the configuration of the explosions may have an effect on Rayleighwave generation.

This dissertation is the result of an investigation of the effect of nuclearexplosion spalling and multipie-explosion configuration on Rayleigh-wave generation.

Chapter II is devoted to an analysis of accelerometer records, to determine how the dimensions of the spall region, the mass of the spalled rock, and the momentum carried by the spall depend on the explosion yield and the depth of burial. Since Eisler and Chilton's (1964) investigation, Perret has accumulated considerably more data, especially on the much higher-yield explosions that were subsequently detonated. He kindly made available surface accelerometer recordings for some of the larger shots whose yields are unclassified. Certain assumptions about the spall process are required to determine mass and momentum from the surface accelerometer records; therefore, the entire explosion and spall phenomena were calculated for a few explosions, by means of numerical modeling techniques, and the results were compared with experimental observations.

Chapter III discusses critical aspects of the numerical methods used in the computer models. The numerical simulation techniques are based on converting the pirtial differential equations descrioing the material's dynamic behavior into algebraic equations suitable for a com- puter. This is done by approximating partial derivatives with finite difference derivatives. A computer program called Tensor (Maenchen and Sack, 1964; Cherry et al., (1971) was developed at the Lawrence Livermore Laboratory to model the dynamic nonlinear, inelastic, and elastic response of earth materials, using finite difference techniques. This protram was used to calculate both the spall phenomena and Rayleigh-wave propagation. The program had not been previously used in any extensive investigation of spall or Rayleigh waves, so it was necessary to mak: some modifications in the program, to analyze certain numerical effects, and to devise a set of test problems to check the program. In particular, thic pisogram contains a numerical term corrmon to many solid mechanics programs, called the linear $Q$, which is added for the purpose of damping out short-wave-length perturbations that cannot be treated accurately by the difference approximation technique. This linear $Q$ was developed empirically, with no consideration of its possible effect on the weak decaying waves of interest in spall or surface wave calculations. Chapter III gives a theoretical analysis of the Linear $Q$.

Chapter IV discusses the comparison of the results from calculation of the entire explosion and spall phenomena with experimental observations. No underground nuclear explosion has ever been monitored with instruments specifically intended to help test the spall theory of Rayleigh-wave generation. Data are available, however, from instruments and measurements included for other purposes A good comparison between numerical calculations of spall 
and experimsntal measurements can be made only if bath accelerometer records and material property measurements are available. Unfortunately there are many shots with extensive accelerometer instrumentation but no measurements of the material properties at the explosion site, and vice versa. This severely limits the number of events useful for comparison tests. Some incomplete material-property are available for two shots, Boxcar and Gasbuggy, which had good surface-motion instrumentation. The surface motion and spall momentum were calculated theoretically for both shots, 12sing the available material property data in a modified version of the Tenisor program. These were compared with the experimental surfacemotion measurements and the spall momentum obtained from the accelerometer records.

Chepter $\mathrm{V}$ applies the results obtained ir the earlier chapters to the Rayleighwave generation problem. Because the implact of the ballistic spall corresponds roughly to a distributed normal load, an approximate analytical solution could be obtained for the resulting Rayleigh wave. Amplitudes calculated from spall impact data were then compared with observed Rayleigh-wave amplitudes. In addition, the numerical techniques described in
Chapter IfI were used to perform a computer experiment. The experiment consists of two runs simulating the same underground explosion with calculation of the resulting Rayleigh waves out to a distance of $20 \mathrm{~km}$. In one run the material response is purely elastic, while, in the other, failure is allowed so that spallation occurs. Comparison of the waves from the two runs allows one to observe the effect of spallation on the Rayleigh wave.

In the final chapter, the effects of simultaneous multiple-explosion configurations and surface topography are examined. The investigation is based on numerical modeling experiments, using the methods described in Chapter III. The model experiments zimulated a single explosion, a vertical line of explosions, and a disk-shaped horizontal array of explosions. Each of these configurations was considered for a free surface in the form of a lat plain, an axially symmetric 2-km-high mountain. and an anially symmetric canyon or crater. All of the runs had the same explosion energy and energy density, so that the amplitudes of the waves could be compared.

The principal results contained in this dissertation have been published in three papers (Viecelli, 1973a, 1973b, 1973c). 


\section{Estimation of Spall Parameters}

\section{A. DISCUSSION}

An important step in testing the spall model of Rayleigh wave generation is to find out if the mass and momentum of the spall material are sufficient to generate Rayleigh waves of the observed amplitude. The spall model assumes that the wave is produced by the impact of the falling spall material. This impact is essentially equivalent to the application of a distributed time-dependent force to the surface. By a simple extension of Lamb's 1904 result, it is possible to calculate the Rayleigh wave for the above source function. The input parameters for this solution are the total impulse, the characterint: time duration, and the area of appication of the force. The impulse delivered by th. impacting spall is just equal to the vertical momentum carried by the spall material. Thus, if the density, the diameter of the circular spall region, the thickness of the spall material, and the velocity at which the spall initially flies up from the surface can be measured, then one can determine the impulse. With this inforration and the measured sound speed, one can also estimate the time duration of the impulse.

Infornation on the dimensions and momentum of the spall matexial as a function of explosion energy and depth of burial is derived from an analysis of surface accelerometer records from the Rainier, Antler, Scotch, Halfbeak, Greeley, and Boxcar Events. The records were made available by Sandia Laboratories, Albuquerque, New Mexico
(Perret, personal communication, 1971). These particular events were chosen out of a much larger population for study because their yields were unclassified, because they were all fired in the same material and in the same area, and because both seismic and accelerometer data were available for each shot. The location, shot time, depth, yield, and other data have been reported by Johnson et al. (1959) and Springer and Kinnaman (1971). Data on yield and scaled depth of burial are given in Table 1.

Table 1. Yield and depth of burial for several nuclear events fired in tuff.

\begin{tabular}{llll}
\hline Event & Date & $\begin{array}{c}\text { Yield } \\
(\mathrm{kt})\end{array}$ & $\begin{array}{c}\text { Scaled depth } \mathrm{si}^{\mathbf{2}} \\
\text { burial } \\
\text { (m/kt }\end{array}$ \\
\hline Rainier & $9 / 19 / 57$ & 1.7 & 227 (204 slant) \\
Antler & $9 / 15 / 61$ & 2.6 & 292 \\
Halfbeak & $6 / 30 / 66$ & 300 & 122 \\
Greel ey & $12 / 20 / 66$ & 825 & 130 \\
Scotch & $5 / 23 / 67$ & 150 & 184 \\
Boxcar & $4 / 26 / 68$ & 1200 & 109 \\
\hline
\end{tabular}

Accelerometer time traces for large expiosions characteristically begin with a positive rectangular pulse followed by a free-fall period of $-1 \mathrm{~g}$, a second positive pulse, and return to $0 \mathrm{~g}$. Although the initial pulse is roughly rectangular, there are fluctuations about the mean. In particular, there is usually a spike on the leading edge; but its width is insignificant, compared to the width of the rest of the pulse. Figure 2 shows a typical accelerometer record. 


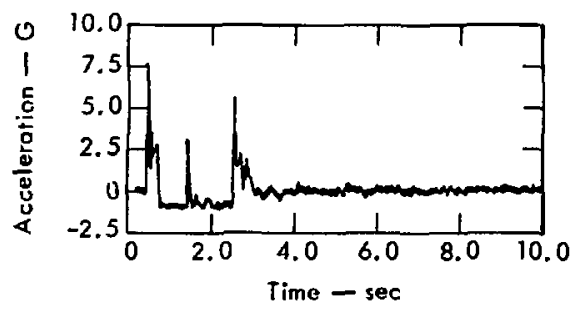

Fig. 2. Vertical accelerometer record for the Boxcar Event. The gage is located at the surface directly above the detonation point.

\section{B. SPALL DIMENSIONS AND VELOCITY}

The parameters needed are the depth, velocity, and radial extent of severe spallation. Estimation of the velocity and radial extent of spallation is straightforward and has been discussed previously by Eisler and Chilton (1964). Estimation of the depth from surface-gage measurements is not so certain and requires some assumptions. The assumptions made are 1) that the decaying shcck wave is roughly symmetrical about its center, 2) that the tensile strength of the earth is negligible, and 3) that the lithostatic pressure can be ignored. Numerical solutions for the decaying spherical shock profile, discussed in the next chapter, show that, in the case of a Voigt solid, the wave approaches a syr netrical Gaussian form. Hence, the as sumption of symmetry is not too badly in error. The second and third assumptions are not so critical, because the pressure in the wave is much larger than both the tensile strength of the rock and the lithostatic preseure near the surface.

With these assumptions, one can make a simple analysis of the spallation process, using linear elastic theory until the instant the material goes into tension. The assumption of small displacements and linear elastic theory in discussing nuclear explosion spall is fairly realistic so long as the analysis is confined to surface material in compression. If thie explosion is buried deeply enough for containment (allowing for a safety factor), the pressure in the initial blast pulse will be only a few hundred atmospheres or less by the time it reaches the surface. By using a triangular pilse and the superposition principle, one inay solve the free-surface interaction problem graphically, as shown in Fig. 3 . The region to the right of the vertical axis represents the earth containing the compression pulse traveling to the left. The wave equation and the f. ee-surface boundary condition of zero pressure may be satisfied by placing a virtual tension wave, propagating to the right, an equal distance to the left of the free-surface line. The solution is the superposition of the incident and virtual pulses. The sequence of solutions in Fig. 3 qualitatively illustrates the spatial variation of the stress and particle velocity as the pulse interacts with the free surface. The acceleration is proportional to the gradient of the stress, so that the surface acceleration time profile corresponding to Fig. 3 is that of a rectangular puise ending at time $t_{5}$. At $t_{5}$ the velocity has reached its maximum value of twice the particle velocity in the incident pulse, and the stresis has vanished, At this instant, spallation occurs. Any continuation of the solution past this time results in a negative, or tension, stress. In this idealized solution the spall has a depth one-half the width of the incident pulse and a velocity profile 


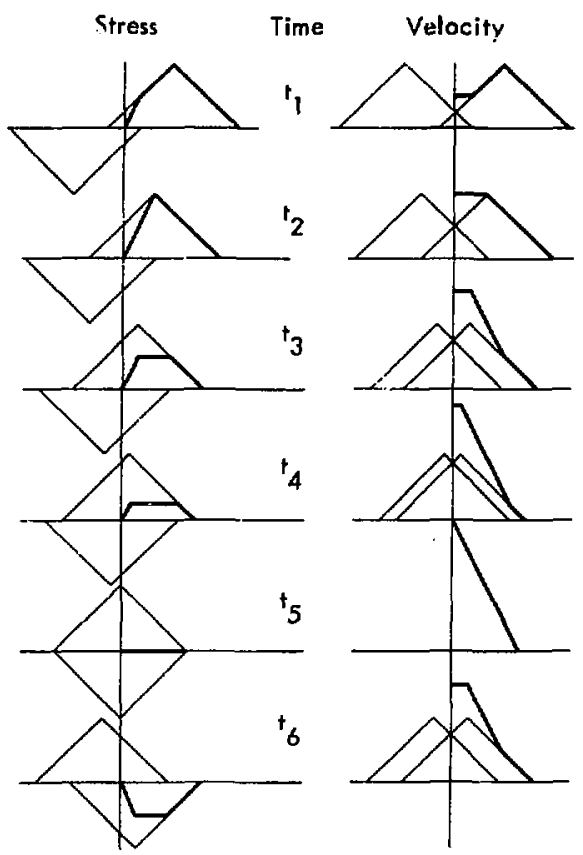

Fig. 3. Grapinical solution for the interaction of a triangular compression pulse with a free surface. The left column shows the stress component parallel to the motion. The stress is positive in com pression, and the wave is shown moving to the left. Tho right column shows the particle velocity. Spallation occurs at time $t_{5}$ for the inelastic case. Time $t_{6}$ shows the tension reflection 'ha: would occur if material did not fail in tension.

that goes from a maximum at the upper surface to zero at the bottom. For purposes of estimating the momentum, this is equivalent to an idealized plate with an effective depth of one quarter of the incident pulse width and a velocity equal to twice the particle velocity in the incident pulse. Since the wave has traveled a distance equal to half its width in the time interval between zero and $t_{5}$, the effective depth is just equal to onehalf the time width of the acceleration pulse times the compressional sound velocity.

Figure 4 shows the effective depth versus the yield for some of the available data. The solid line running through the data has a slope of one-third, corresponding to one-third power law scaling. This might be expected, because the depth of spallation depends on the amplitude and spatial extent of the explosion shock, which, in turn, scales as the one-third power of the yield. For one-third power law scaling to hold exactly, each event would have to be in the same material at the same scaled depth, and gravitational effects would have to be negligible. The maximum variation in the scaled depth of the data points is about a factor of 3 , which apparently accounts for the tendency to a slope greater than one-third. One must also realize that the inethod of estimating the depth from the accelerometer is fairly crude.

Compared to these two sources of scatter, the gravitational effect is small.

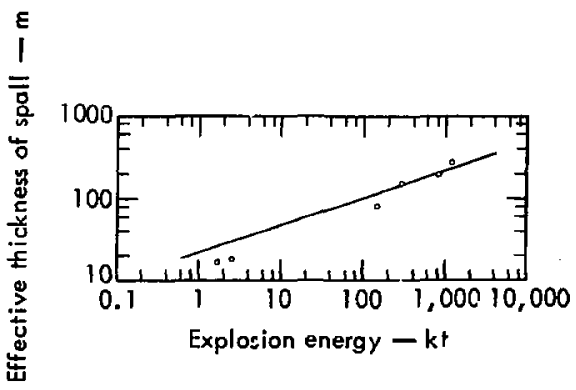

Fig. 4. Estinated effective spall thickness plotted against explosion energy release $w$ thout regard for variation in depth of burst. 
The effect of gravitation is to praduce a density and strain energy gradient in the earth. This, in turn, causes the portion of the shock traveling vertically to decay at a slightly slower rate than it otherwise would, The effect is similar to that which occurs for a shovk propagating vertically in an exponential atmosphere ( $\mathrm{Zel}$ 'dovich and Raizer, 1966-1967), except that it is much less pronounced because of the high density and stiffness of the earth. The main factor is the characteristic of the material surrounding the shot. The porosity and water content of the rock affect the strength of the shock transmitted to the surface and have a strong influence on scatter in the data, All of the shots were fired in tuff within a few tens of miles of each other.

From one-third power law scaling, one would expect that spall depths scaled by the third power of the yield should be the same if the scaled depth of burial and the material properties are the same. Therefore, by plotting scaled spall thickness versus scaled depth of burial, one should obtain an indication of the dependence of spall thickness on depth, as shown in Fig. 5. The solid line corresponds to an inverse first-power law. Assuming that spall thickness varies inversely with the shot depth, one can adjust the data in Fig. 4 to correspond to the same scaled depth of burial. For example, to adjust the Antler data in Fig. 4 to correspond to the same scaled depth of burial as Halfbeak $\left(122 \mathrm{~m} / \mathrm{kt}^{1 / 3}\right)$, one would multiply the Antler spall thickness by 292/122. The adjusted data on spall thickness, shown in Fig. 6, are seen to be in much closer agreement with a one-third power law dependence on yield.

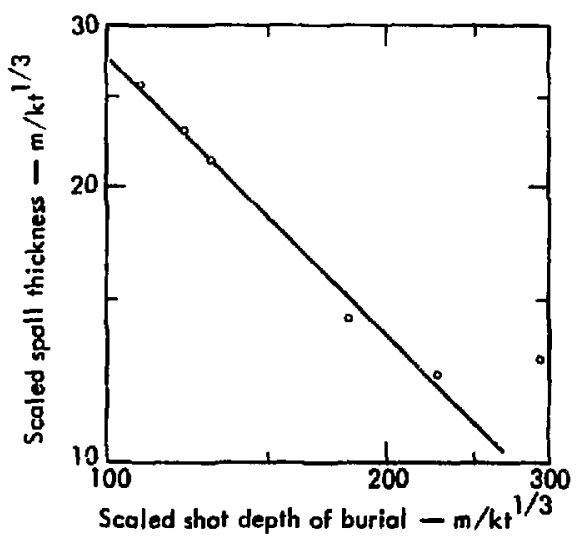

Fig. 5. Scaled spall thickness plotted against scaled shot depth of burial. The solid line corresponds to an inverse first -power iaw.

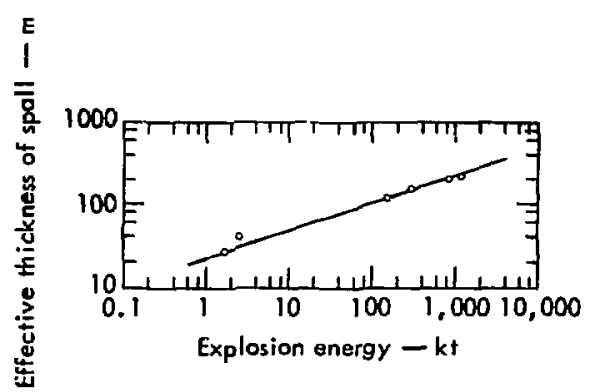

Fig. 6. Adjusted spall thickness plotted against explosion energy release. The solid line has a slope of onethird. All shots in tuff.

The method used by Eisler and Chilton (1964) to estimate the radial extent of the spall is to plot the free-fall time versus range for a set of acc slerometers along a radius from ground zero. The region of severe spallation is assumed to extend to a range at which the free-fall time is small and slowly varying. Figure 7 shows the radial extent of the spall versus 


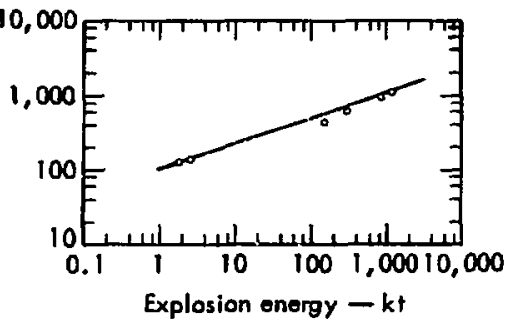

Fig. 7. Radius of severe spallation plotted against explosion energy release. The line has a alope of one-third.

yield estimated by this method. Figure 8 shows the scaled spall radius versus the scaled depth of burial. The solid line in Fig. $\theta$ is a least squares fit to the tuff data. The weak dependence of the scaled spall radius on the scaled depth of burial appears to indicate that increased spallation with decreasing depth is approximately balanced by decreased spallation with increasing angle of incidence of the wave.

\section{MASS AND MOMENTUM OF SPALL}

It follows from the one-third power law dependence of depth and radius that the volume of spalled material, and hence the mass, is proportional to the yield for shots at the same scaled depth of burial. By means of the curves in Figs. 6 and 7, one obtains the following rough estimate for the effective spall mass of shots fired in tuff (density $\approx 2.0 \mathrm{~g} / \mathrm{cm}^{3}$ ):

$$
\langle\mathrm{M}\rangle \approx 1.6 \times 10^{12} \mathrm{~W} \text { grams }
$$

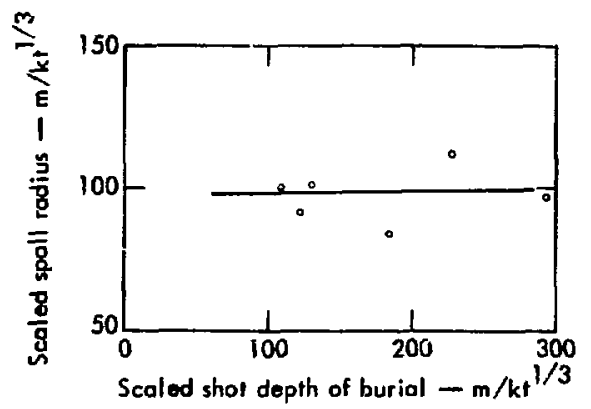

Fig. 8. Scaled spall radius plotted against scaled shot depth of burial. The line is a least-square fit.

where $W$ is the explosion energy in kilotons $\left(1\right.$ kiloton $=10^{12}$ calories) and the scaled depth of burial is $122 \mathrm{~m} / \mathrm{kT}^{1 / 3}$.

The momentum trapped within the spall may be estimated from experimental measurements of maximum surface displacement. From their study of accelerometer data, Eisler and Chilton (1964) concluded that the suriacedisplacement profile is conical with sides concave upward. The data from the larger shots also exhibit this roughly conical, concave-up profile. For estimating the momentum, the surface displacement is reasonably well approximated by a quadratic function of the distance from the spall radius, with maximum displacement at ground zero and zero displacement at the spall radius.

The maximum ground-zero surface displacement is plotted against scaled depth of burial in Fig. 9. The data exhibit an inverse fourth-power dependence on depth of burial. This dependence can be explained by the fact that the height attained by the free-falling spall is a 


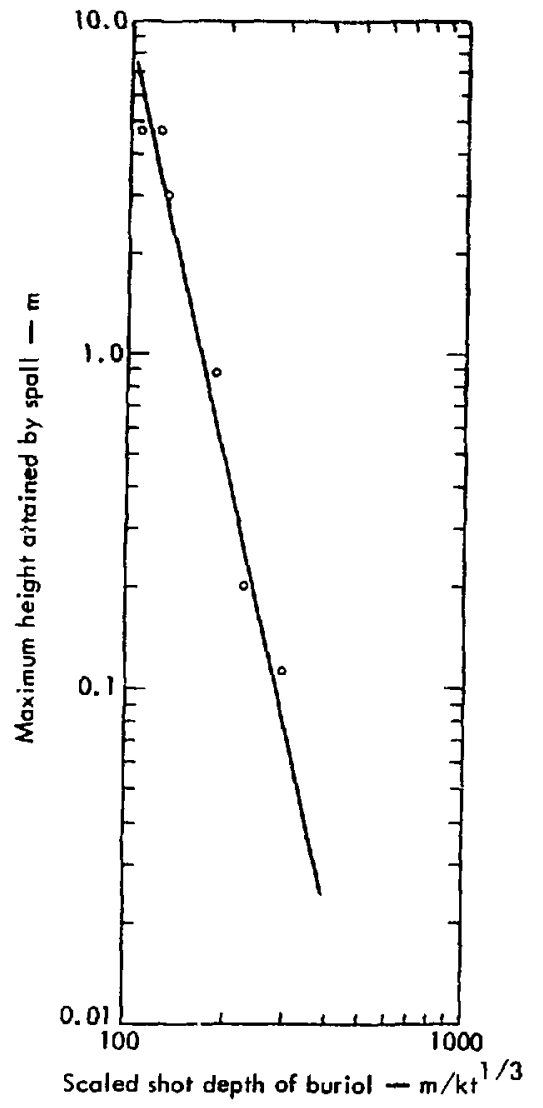

Fig. 9. Maximum height attained by ballistic spall plotted against scaled shot depth of burial.

measure of the particle velocity of the expiosion shock. This follows because the height attained in free fall is proportional to the square of the spall velocity. The spall velocity, in turn, is equal to twice the particle velocity in the incident shock. An inverse-square law of deci.y for the shock particle-velocity therefore produces an inverse fourth-power dependence of maximum surface displacement on depth of burial. The maximum surfacedisplacement data for tuff therefore indicate that the shock particle-velocity is following an inverse-square decay. If no other attenuating mechanisms, other than spherical spreading, were present, the shock would follow an inverse first-power decay. The higher decay rate observed in the tuff data indicates the presence of other attenuating mechanisms in addition to geometrical spreading.

To estimate the total momentum trapped in the spall plate, solve for the velocity profile consistent with the conical displacement profile, multiply by the density, and integrate over the volume of the plate. I is used to denote this quantity, since it also represents the downward impulse delivered to the earth's surface when the spall plate falls back.

The connection between velocity and displacement is obtained by equating the maximum gravitationai potential energy to the initial kinetic energy of the spall plate:

$$
(1 / 2) v^{2}=g h=g h_{o}\left(1-r / r_{o}\right)^{2},
$$

where $h_{0}$ is the maximum height attained by the spall plate and $r_{0}$ is the radius of the plate. Therefore,

$$
\begin{aligned}
I=\rho \mathrm{d}_{\mathrm{o}}\left(2 g \mathrm{~h}_{o}\right)^{1 / 2} & \int_{0}^{r_{0}} 2 \pi r\left(1-r / r_{o}\right) d r \\
& =(\pi \beta)_{\rho} \mathrm{d}_{\mathrm{o}} \mathrm{r}_{\mathrm{o}}^{2}\left(2 g \mathrm{~h}_{\mathrm{o}}\right)^{1 / 2} .
\end{aligned}
$$

Since $\mathrm{n}_{\mathrm{o}}$ measures the shock particle velocity, it is dependent only on the scaled depth of burial. Thereiore, at a fixed scale depth of burial the impulse is proportional to the mass, which, in turn, 
is proportional to the explosion energy

W. From (1) and the plot of $h_{0}$ in Fig. 0 , we find that, at a scaled depth of burial of $122 \mathrm{~m} / \mathrm{kT}^{1 / 3}$ in tuff.

$$
\langle l\rangle=4.6 \times 10^{14} \mathrm{~W} \text { dyne sec, }
$$

where $W$ is the explosion energy release in kilctons.
Some feeling for the acale involved can be conveyed by giving the data for the Boxcar event in ereryday units. The explosive energy release was 1.2 Mt at a depth of $3800 \mathrm{ft}$. This propelled a slab of Nevada tuff, about 1.2 miles in diameter and $750 \mathrm{ft}$ thick, weighing approximately 2 billion tons, to a height of $15 \mathrm{ft}$ at the center tapering to $1 \mathrm{ft}$ at the edge. 


\section{Numerical Methods}

\section{A. DISCUSSION}

The spall phenomenon can algo be calculated by computer programs designed to solve solid mechanics problems involving explosive loading. These programs compute the time-dependent response of the material in two or three spatial dimensions with axial symmetry. They allow nonlinear response, crushing of the material, plastic flow, and, in some versions, brittle fracture. The numerical simulation techniques are based on converting the partial differential equations describing the physics into algebraic equations suitable for a computer. This is done by approximating the derivatives by finite differences. Usually the finite difference equations are written in the Lagrangian coordinate frame so that the computer programs are extremely flexible and adaptable with respect to the geometrical shapes, the boundary conditions, and the kinds of different material regions that may be included in a problem. Because of this tremendous flexibility and comprehensiveness, it is often possible to model most of the significant aspects of an actual explosion experiment. The use of such programs is, in fact, much more similar to the performance of an actual experiment than to the derivation of a mathematical result. Although every aspect of the conditions describing the numerical experiment are known and can be varied at will, each computer run can give the results for only one specific vase. The interpretation of these results can be difficul+ if the program models a large number of physical phenomena. Furthermore, spurious results, analogous to experimental errors, can be caused by the numerical approximation techniques or by program instruction errors. Before proceeding with any silidy based on numerical simulation experiments, one must carefully analyze the numerical techniques uded, to make certain that they are accurate for the class of problems to be studied.

This chapter examines two problems of numerical accuracy. The Tensor program was used in this analysis. It was originally developed by Maenchen and Sach (1964) and then modified by Cherry et al. (1971). The version published by Cherry has been modified for use herein. These modifications will be described in this chapter and Chapter IV. Except as they relate to these modifications, the details of the Tensor program will not be discussed, since they are presented in the references cited. The first objective of the numerical analysis study has been to determine what effect the linear artificial viscosity normally used in the program may have upon the accuracy of a spall calculation. The first half of this chapter presents an analysis of this problem. The second abjective has been to make certain that the Tensor program would accurately compute Rayieigh-wave propagation, and to determine the limitations on accuracy imposed by numerical truncation and artificial viscosity. The last half of the chapter discusses the analysis of this problem. 


\section{B. ANALYSIS OF ARTIFICIAL VISCOSITY}

A characteristic of an explosion contained by a solid, in this case the earth, is that the pressure and particle velocity decay rapidly with increased distance from the detonation point. Not far from the explosion, the material response of the medium is nearly linear in the sense that the convective terms in the Eulerian description of momentum conservation are small compared with the pressure terms. Blast waves that satisfy this condition are called weak decaying shocks throughout the rest of this chapter.

Solid mechanics programs integrate the following Lagrangian equation:

$$
\begin{aligned}
\frac{\partial u}{\partial t}=-\frac{1}{\rho} \frac{\partial P}{\partial R}+\frac{1}{\rho} \frac{\partial \tau_{R Z}}{\partial Z}+\frac{1}{\rho} \frac{\partial \tau_{R}}{\partial R} \\
+\frac{2 \tau_{R}+\tau_{Z}}{\rho R}+G_{R}
\end{aligned}
$$

$$
\begin{aligned}
\frac{\partial v}{\partial t}=-\frac{1}{\rho} \frac{\partial P}{\partial Z} & +\frac{1}{\rho} \frac{\partial \tau R Z}{\partial R} \\
& +\frac{1}{\rho} \frac{\partial \tau_{Z}}{\partial Z}+\frac{\tau R Z}{\rho R}+G_{Z}
\end{aligned}
$$

$$
\frac{\partial \tau}{\partial t}=\frac{2 \mu}{3}\left(2 \frac{\partial u}{\partial R}-\frac{\partial v}{\partial Z}-\frac{u}{R}\right)
$$

$$
\frac{{ }^{\partial \tau} z}{\partial t}=\frac{2 \mu}{3}\left(2 \frac{\partial v}{\partial z}-\frac{\partial u}{\partial R}-\frac{u}{R}\right)
$$

$$
\frac{{ }^{\partial \tau} R Z}{\partial t}=\mu\left(\frac{\partial u}{\partial Z}+\frac{\partial v}{\partial R}\right)
$$

$\frac{\partial \mathbf{R}}{\partial t}=u$

$$
\frac{\partial Z}{\partial t}=v .
$$

Iere $\mathrm{R}$ is the radial coorclinate, $\mathrm{Z}$ is the axial coordinate. ' 2 is the radial component of velocity, $v$ is the axial component of velocity, $t$ is the time, $\rho$ is the density, $P$ is the pressure, $G_{R}$ and $G_{Z}$ are the components of gravitational acceleration, ${ }^{\tau} \mathbf{R},{ }^{\tau} Z$, and $\tau_{\mathbf{R Z}}$ are the deviatoric stresses, and $\mu$ is the shear modulus.

The equation of state is usually in the form

$$
\mathrm{P}=\mathrm{f}(\theta), \quad \theta=\frac{\rho}{\rho_{\mathrm{o}}}-1 .
$$

The density of a zone in the finite difference approximation is determined by dividing the mass of the Lagrangian zone (which remains constant throughout the calculations) by the volume of the zone, which, in turn, is calculated from the coordinates of the mesh points defining the zone at the current time.

$Y$ ield and failure are incorporated by altering the deviatoric stresses $\tau_{R}$ ' $\tau_{Z}$, ${ }^{T} \mathbf{R Z}$ at each time step of the integration, according to a prescription based on the current state of stress. In the case of elastic behavior, the stresses are left unaltered and the pressure is computed from

$$
\mathbf{P}=\mathrm{k} \theta,
$$

where $\mathrm{k}$ is the bulk modulus.

The computer programs for solving these equations (in addition to Tensor, see also Wilkins, 1964, 1969) rely on different finite difference approximations to the above partial differential equations. However, they have one feature common to all elastic-plastic Lagrangian difference codes: the substitution of $F+Q$ for $P$ where $Q$ is the "ps eudo-viscosity" 
introduced by Von Neumann (see Richtmyer and Morton, 1967). The purpose of $Q$ is to provide a mechanism by which kinetic energy can be converted to internal energy as the material is shocked, In the case of motion in one spatial dimension, the quadratic $Q$ is intended for very strong shock waves and has the form

$$
Q=\left\{\begin{array}{ll}
C_{0} \rho(\Delta x)^{2}\left(\frac{\partial u}{\partial x}\right)^{2}, & \frac{\partial u}{\partial x}<0 \\
0, & \frac{\partial u}{\partial x}>0
\end{array},\right.
$$

where $\rho$ is the density, $C_{0}$ is a constant of the order of 1, and $\Delta x$ is the size of the spatial difference interval.

As noted earlier, the spherical shock wave from an explosion in a solid decays rapidly with distance, As a result, one quickly reaches a range at which quadratic, or second-order, terms become small, compared with the îirst-order terms in the difference equations of motion. To counteract this, investigators have added linear or first-order terms to the expression for $Q$. There have been many formulations of $Q$, but a fairly typical example in one spatial dimension is

$Q=\left\{\begin{array}{ll}C_{0} \rho(\Delta x)^{i}\left(\frac{\partial u}{\partial x}\right)^{2}-C_{1} \rho \alpha \Delta x\left(\frac{\partial u}{\partial x}\right), & \frac{\partial u}{\partial x}<0 \\ -C_{1} \rho \alpha \Delta x\left(\frac{\partial u}{\partial x}\right) & , \frac{\partial u}{\partial x}>0\end{array}\right.$.

where $C_{1}$ is a constant, $a$ is the compressional sound velocity, and $\Delta \mathrm{x}$ is the mesh width. As the wave decays, the linear terms in this $Q$ remain comparable to the other terms in the equations of motion and continue to perform the function of converting the kinetic energy of oscillation between neighboring mesh points into internal energy.

In fact, the inclusion of linear $Q$ term amounts to adding dissipation terms to the basic differential equations. If one defines the viscosity coefficient $\eta \equiv C_{1}$ $\rho a \Delta x$ and takes the limit $\Delta t \rightarrow 0, \Delta R \rightarrow 0$, $\Delta Z \rightarrow 0$ while holding $\eta$ constant, the difference equations approach the differential equations for the voigt-Kelvin model of a viscoelastic solid. In practice, one almost always operates with a value of $C_{1}$ such that $\eta$ is large enough that the $Q$ terms dominate any of the truncation effects arising from the difference approximation to the other terms in the differential equations. For this reason, the solutions obtained from the difference equations approach very closely the solutions one obtains for the differential equations with dissipation terms added.

The intent of this analysis is to provide a quantitative understanding of the effect of the linear $Q$ upon the attenuation of a spherical shock. Because the phenomenon is so nearly linear, except in the immediate neighborhood of the explosion, it is easier to attack the problem at the level of the partial differential equations rather than that of the difference equations. The critical role $Q$ plays in determining the pulse shape and particle velocity in a weak spherical shock will be demonstrated, and it will be shown that the presence of a linear $Q$ implies an inverse three-halves power decay law.

With the addition of the linear $Q$ used in my version of the $x$ ensor program, the differential equations become 


$$
\begin{aligned}
\frac{\partial u}{\partial t}= & -\frac{1}{\rho} \frac{\partial\left(P+Q-\tau_{R}-Q_{R}\right)}{\partial R} \\
& +\frac{1}{\rho} \frac{\partial\left(\tau_{R Z}+Q_{R Z}\right)}{\partial Z}- \\
& +\frac{2 \tau_{R}+\tau_{Z}+2 Q_{R}+Q_{Z}}{\rho R}
\end{aligned}
$$

and

$$
\begin{aligned}
\frac{\partial v}{\partial t} & =-\frac{1}{\rho} \frac{\partial\left(P+Q-\tau_{Z}-Q_{Z}\right)}{\partial Z} \\
& +\frac{1}{\rho} \frac{\partial\left(\tau_{R Z}+Q_{R Z}\right)}{\partial R}+\frac{{ }^{\top} R Z+Q_{R Z}}{\rho R}
\end{aligned}
$$

where $\tau_{R}, \tau_{Z}$, and $\tau_{R Z}$ are the deviatoric stresses, and the $Q, Q_{R}, Q_{Z}$, and $Q_{R Z}$ are given by

$$
\begin{aligned}
& Q_{R}=\frac{2 \eta}{3}\left(2 \frac{\partial u}{\partial R}-\frac{\partial v}{\partial Z}-\frac{u}{R}\right) \\
& Q_{Z}=\frac{2 \eta}{3}\left(2 \frac{\partial v}{\partial Z}-\frac{\partial u}{\partial R}-\frac{u}{R}\right) \\
& Q_{R Z}=\eta\left(\frac{\partial u}{\partial Z}+\frac{\partial v}{\partial R}\right) \\
& Q=-\lambda\left(\frac{\partial v}{\partial Z}+\frac{1}{R} \frac{\partial u R}{\partial R}\right) . \\
& \eta=C_{1} \rho \alpha\langle\Delta x\rangle \\
& \lambda=C_{1} \rho \alpha\langle\Delta x\rangle .
\end{aligned}
$$

Here $\langle\Delta x\rangle$ is the characteristic mesh length and $C_{1}$ is an input constant. In a two-dimensional calculation, $\langle\Delta x\rangle$ is determined from a weighted average of the distances between the mesh points defining a zone. In one-dimensional calculations, $\langle\Delta x\rangle$ is just the width of a zone. Although $\eta$ and $\lambda$ are numerical and not physical quantities, the notation common to denote shear viscosity and bulk viscosity has been used to emphasize the equivalence of the linear $Q$ formulation to the Voigt-Kelvin model of a viscoelastic solid.

The deviatoric stresses are determined by the Eqs. (7), (8), and (9). With the assumption of linearity, the spatial differentials with respect to the Lagrangian coordinates may be replaced by spatial differentials with respect to Eulerian cocrdinates. Substitution of Eqs . (10) and (11) into Egs. (7), (8), (9), (18), (19), (20), and (21) and, in turn, substitution of these equations and Eq. (13) into Eqs. (16) and (17) results in a pair of linear partial differential equations for the Lagrangian displacements $\Delta R$ and $\Delta Z$. In the theory of elasticity it is shown that the elastic displacement equations can be further simplified by the introduction of scalar and vector potentials. In the case of spherical symmetry the vector potential is zero, and one need only solve for the scalar displacement potential given by

$$
\begin{aligned}
\frac{\partial^{2} \phi}{\partial t^{2}}= & \left(\frac{k+\frac{4 \mu}{3}}{\rho}\right) \frac{1}{r^{2}} \frac{\partial}{\partial r}\left(r^{2} \frac{\partial \phi}{\partial r}\right) \\
& +\left(\frac{\lambda+\frac{4 \eta}{3}}{\rho}\right) \frac{1}{r^{2}} \frac{\partial}{\partial r}\left(r^{2} \frac{\partial^{2} \phi}{\partial r \partial t}\right) .
\end{aligned}
$$

The radial displacement is then given by

$$
\Delta \mathbf{R}=-\frac{\partial \phi}{\partial \mathbf{r}} .
$$

The velocity potential is given by

$$
\Phi=\frac{\partial \phi}{\partial t},
$$

so that the particle velocity is given by

$$
\mathbf{u}=-\frac{\partial^{2} \phi}{\partial \mathbf{r} \partial t} \text {. }
$$


In deriving the basic differential equation corresponding to the spherical shock problem with the linear $Q$, the coefficients $\eta$ and $\lambda$ have been taken to be constants, whereas in the standard solid mechanics programs they usually have some spatial variation as a result of variations in the mesh width, density, and sound speed. Furthermore, the linear $Q$ is assumed effective in both compression and expansion regions, whereas in the traditional formulations the linear $Q$ is set to zero in expansion regions. My program has constant input values for $\eta$ and $\lambda$ and leaves the linear $Q$ on in expansion regions, so that all of the requirements for the analytic solution are strictly satisfied.

The driving mechanism for the linear problem that approximates the explosion closely at early times is that of a small cavity expanding at constant velocity. Analytically this is represented by a boundary condition of the form

$$
\operatorname{limit}_{r \rightarrow 0} \phi=\frac{\phi_{0} t}{r} .
$$

To solve for the asymptotic form of the decaying spherical shock with linear $Q$, put $\phi=\Lambda / \mathrm{r}$ and substitute into Eq. (24) to get

$$
\frac{\partial^{2} \Lambda}{\partial t^{2}}=\alpha^{2} \frac{\partial^{2} \Lambda}{\partial r^{2}}+\nu \frac{\partial^{3} \Lambda}{\partial r^{2} \partial t},
$$

where

$$
\alpha^{2}=\frac{k+\frac{4}{3} \mu}{\rho}
$$

and

$$
\nu=\frac{\lambda+\frac{4}{3} \eta}{\rho} .
$$

Taking the Laplace transform $H$ of $\Lambda$ with respect to time gives

$$
\begin{aligned}
\mathrm{s}^{2} \mathrm{H}-\mathrm{s} \Lambda(0)-\dot{\Lambda}(0) & =a^{2} \frac{\partial^{2} \mathrm{H}}{\partial \mathrm{r}^{2}} \\
& +\nu \frac{\partial^{2}}{\partial \mathrm{r}^{2}}\{\mathrm{sH}-\Lambda(0)\} .(32)
\end{aligned}
$$

Since the material is initially at rest, $\Lambda$ and $\dot{\Lambda}$ are identically zero:

$$
\frac{d^{2} H}{d r^{2}}-\left(\frac{s^{2}}{\alpha^{2}+\nu s}\right) H=0 .
$$

The solution, which decays to zero as $r \rightarrow \infty$, is

$H(r, s)=A(s) \exp \left[-r s\left(\alpha^{2}+\nu s\right)^{-1 / 2}\right]$.

The Laplace transform of the boundary condition at $r=0$ is

$$
L\left(\phi_{0} t\right)=\phi_{o} s^{-2}
$$

Therefore, the transform of the solution is

$$
\begin{aligned}
H(r, s)= & \phi_{0} s^{-2} \\
& \times \exp \left[-r s\left(\alpha^{2}+\nu s\right)^{-1 / 2}\right] .
\end{aligned}
$$

Taking the time derivative of the solution corresponds to multiplying the transform of the solution by $s$, since $\Lambda(+0)=0$; therefore, the transform of $\dot{\Lambda}$ is

$$
\begin{aligned}
F(r, s)= & \phi_{0} s^{-1} \\
& \times \exp \left[-r s\left(\alpha^{2}+\nu s\right)^{-1 / 2}\right]
\end{aligned}
$$


The inversion may be performed easily when

$$
\frac{r \alpha}{2 \nu} \gg 1,
$$

corresponding to the asymptotic limit to give the velocity potential

$$
\phi(r, t)=\dot{N} / r=\frac{\phi_{0}}{2 r}[1+\operatorname{er} f(\tau \sqrt{\sigma})],
$$

where

$$
\tau=\frac{\alpha \mathrm{t}}{\mathrm{r}}-1
$$

and

$$
\sigma=\frac{r \alpha}{2 \nu}
$$

Therefore, the solution for the particle velocity is

$$
\begin{aligned}
u=\frac{\phi_{0}}{2 r^{2}} & {[1+\operatorname{erf}(\tau \sqrt{\sigma})} \\
& \left.+(2+\tau)\left(\frac{\sigma}{\pi}\right)^{1 / 2} \exp \left(-\sigma \tau^{2}\right)\right] .
\end{aligned}
$$

The analytic solution has been evaluated for a particular case, and the results are plotted as small circles in Fig. 10.

The parameters for thie sase are $\phi_{0}=1.6$ $\times 10^{8} \mathrm{~cm}^{3} / \mathrm{sec}, \nu=7.5 \times 10^{7} \mathrm{~cm}^{2} / \mathrm{sec}$, $\alpha=2.3 \mathrm{~km} / \mathrm{sec}$, and $t=0.35 \mathrm{sec}$. Figure 10 also includes the results for a Tensor calculation of the same problem. These are plotted as small crosses. The elastic constants enter into the analytic solution only by way of the compressional sound velocity; thus there are two additional variables in the finite-difference problem. These are the density and Poisson's ratio, which were chosen to be $2.0 \mathrm{~g} / \mathrm{cm}^{3}$ and $1 / 3$, respectively. The

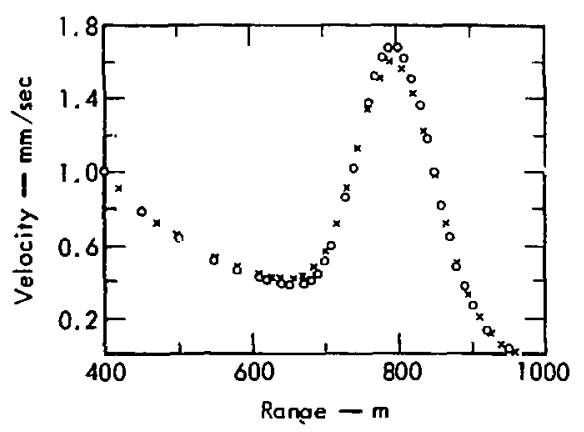

Fig. 10. Shock particle velocity plotted as a function of range. The small circles are points evaluated from ti:e analytic solution. The crosses are points obtained from a finite-difference solution. The time is $0.35 \mathrm{sec}$.

choice is arbitrary, since, once these two variables are fixed, the bulk modulus and shear modulus are determined by the suind velocity.

Spierical symmetry was simulated in the numerical problem by choosing a weage-snaped mesh with one edge along the $Z$ axis of axial symmetry and the other edge at an angle of $10^{\circ}$ from the $Z$ axis. The wedge began at an inner radius of $20 \mathrm{~m}$ and extended out to $1150 \mathrm{~m}$. The analytic result is the asymptotic solution for a cavity expanding at constant velocity. Thus it is necessary to start the numerical calculation with a cavity small enough to ensure that the asymptotic state is reached before results are compared. Furthermore, the cavity must not be driven with too large a velocity, or the linearity assumption would be violated. These precautions must be taken to guarantee that the source function in the numerical probiem is well-defired in terms of the source in the analytical solution. 
It should be emphasized that the asymptotic state is eventually reached, whether or not the linearity assumption is violated in the neighborhood of the cavity. For a nonlinear source, there is always some particular amplitude for the linear source that produces the same asymptotic state as the original nonlinear source. The precautions mentioned ensure that the numerical problem starts with the equivalent linear source amplitude known beforehand. The cavity velocity corresponding to the value of $\phi_{0}$ in the analytical solution is $40.0 \mathrm{~cm} / \mathrm{sec}$. That value satisfies the analytical boundary condition given by Eq. (28) for an initial cavity radius of $20.0 \mathrm{~m}$.

The numerical calculation was done on a mesh containing 140 radial zones. The zoning was not uniform, so as to permit relatively fine zoning next to the cavity. This is necessary to get a good approximation to the analytical source at zero radius. The slight amplitude discrepancy observed in Fig. 10 is the result of this approximation. This was confirmed by making several runs, each with a smaller driving cavity and finer zoning. The amplitude of the numerical solution was observed to converge to the analytical result as the resolution in the cavity retion increased. The zone size at the cavity for the run in Fig. 10 was $2.0 \mathrm{~m}$. The zone size increased gradually to $15.0 \mathrm{~m}$ at a radius of $550.0 \mathrm{~m}$. Beyond this radius the zone size remained constant at $15.0 \mathrm{~m}$.

By referring to Eqs. (22), (23), and (31), one can see that beyond $550 \mathrm{~m}$ the zone size and the parameters $\nu$ and $\alpha$ are equivalent to a linear $Q$ coefficient of 0.1 , $\checkmark$ ich is the value normally used in
Tensor calculations. The fact that the computer solution continues to agree with the ana:ytical solution beyond $550 \mathrm{~m}$ supports the contention that the linear $Q$, as actually used in practical calculations, dominates truncation effects arising from other terms in the equations. To check this, the free surface was moved out to $2005 \mathrm{~m}$ (with the same 15.0-m zone size), and the calculation continued until the pulse reached $1500 \mathrm{~m}$. At the greater range, the pulse approaches a symmetrical Gaussian form similar to the example shown in Fig. 11, but the close agreement between computer and analytical solutions is mairtained.

An indication of how closely an actual explosion calculation approaches the asymptotic form imposed by the linear $Q$ is given by the particle velocity plot in Fig. 11. The Tensor finite difference results are indicated by the small crosses,

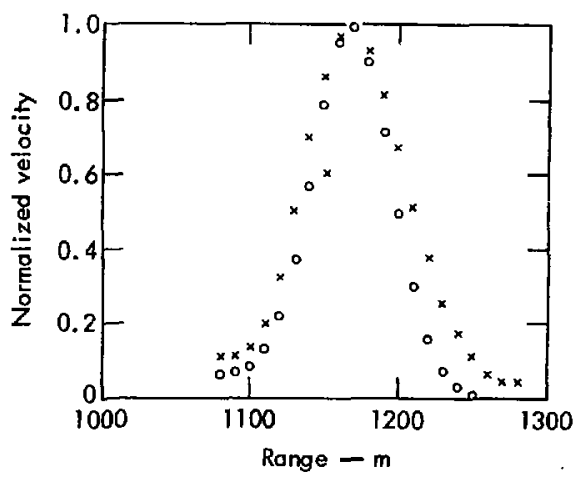

Fig. 11. Normalized shock particle velocity plotted as a function of range. The small circles are points evaluated from the analytic solution, and the crosses are points obtained from the finitedifference solution. The time is $0.375 \mathrm{sec}$. 
and the asymptotic solution by the small circles. The numerical calculation simulated a 25.0-kt explosion in the earth at a depth of $1280 \mathrm{~m}$. The source was a spherical cavity, initially with a radius of $5.0 \mathrm{~m}$, containing vaporized rock at a pressure of $1.74 \mathrm{M}$ bar. The gas expanded adiabatically, forcing the walls of the cavity out to a radius of approximately $30 \mathrm{~m}$. The containing rock was programmed to have a shear strength of only a few kilobars; therefore, severe plastic flow occurred. In addition, the numerical simulation included overburden and several different layers of earth and rock materials, so that the portion of the shock propagating vertically passed through a density and strain energy gradient. It is not necessary to go farther into the details of the numerical run except to note that the average values of $\alpha$ and $\nu$ were $3.12 \times 10^{5} \mathrm{~cm} / \mathrm{sec}$ and 1.85 $\times 10^{7} \mathrm{~cm}^{2} / \mathrm{sec}$, respectively. These values were used in evaluating the analytical solution. The plot shown in Fig. 11 was taken along a radius, which extended vertically to the surface, located at $1280 \mathrm{~m}$. The failure of the numerical solution to go to zero on the leading side of the pulse is explained by the fact that the pulse is just beginning to strike the surface. Despite all of the nonlinearities and inelastic behavior in the source region, the resulting blast wave is in close agreement with the predictions of the asymptotic linear theory.

The development and discussical has been based on a formulation of the linear $Q$ that is active in both compression and expansion zones; however, most investigators simply turn the linear $Q$ off in expansion regions, maintaining that there should not be a dissipative mechanism there.

To examine this case, the first problem (the run shown in Fig. 10) was rerun with the $Q$ terms set to zero in expansion regions. Figure 12 is a duplicate of Fig. 10, with the addition of the run with the $Q$ turned off in expansion. It can be seen that this run is the same as the firs* except in the expansion region, where the removal of the linear $Q$ has introduced an osrillatory perturbation. The decay rate for this run was observed to follow an inverse 1.5-power law,

The intent of this work has been to gain a theoretical understanding of the linear $Q$ and its effect on a weak blast pulse in a solid. The quadratic $Q$ was intended as a convenient computational device for including strong shock discontinuities in inviscid gas-dynamic calculations. Since the physical model assumed inviscid flow, it was natural to suppress viscosity effects everywhere except at a shock. One of the main reasons for the

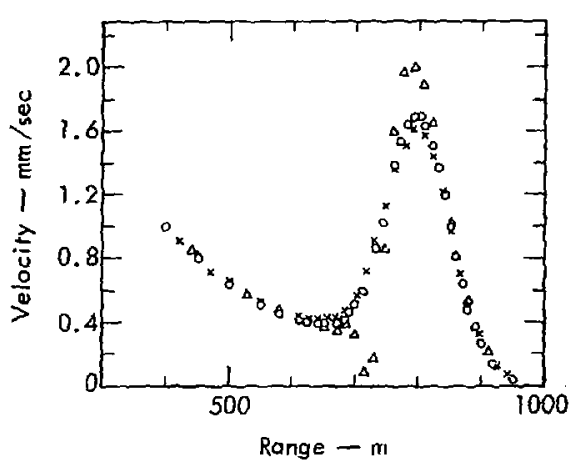

Fig. 12. Duplicate of Fig. 10, with the addition of a run with the linear $Q$ set to zero in expansion regions. Data points from this run are indicated by the small triangles. The time is 0.35 sec. 
subsequent development of the linear $Q$ is the fact that, unless some kind of linear damping is included in the calculation of weak decaying waves, the solutions quickly become obscured in a hashwork of short-wave-length noise. The idea that viscosity must be suppressed except at the shock front has persisted through the development of the linear $Q$. first in applications to gases and then finally to solids.

The underlying physics of weak decaying shocks in solids, and in gases also, is different from that of high-pressure, high-speed gas dynamics. In the latter case, viscous effects can usually be ignored except in shocks or thin boundary layers. In the former case, at some point in the decay of the shock pulse, this physical attenuation mechanisms come to have a dominant effect on the pulse form and decay rate. A comprehensive discussion of the shock decay process is given by Lighthill (1956). The shock transition region thickens until there is no recognizable shock, just a smoothly varying attenuated sound pulse. In solids and earth materials this point may be very near the site of the explosion. For example, from the results of Chapter II one can see that the shock fronts from large nuclear explosions in Nevada tuffs are spread over as much as several hundred meters by the time the front has traveled a kilometer. It is at this finite-amplitude sound-wave stage that numerical noise becomes a problem unless suppressed by some kind of linear damping mechanism.

In practice the solid mechanics programs cannot compute purely elastic response because the calculations must always be made with a finite number of zones. In the case of decaying explosion waves, what they do calculate is the response for a type of Voigt solid, but with viscosity coefficients that depend on purely numerical parameters. The fact that some type of viscosity is required for an adequate physical model of decaying shocks suggests that the use of the linear $Q$ could be made more rational. This can be done by making it correspond exactly to some physical model of damping, rather than having it depend on zone size and shape and on whether or not the zone is in expansion or compression. The easiest thing to do is to make the linear $Q$ correspond exactly to the Voigt model by keeping the $Q$ on in both compression and expansion and by making the coefficients $\eta$ and $\lambda$ in Eqs. (18) through (21) input viscosities. My version of the Tensor program incorporates these changes.

It is evident from the preceding results that the amplitude and shape of the blast pulse are strongly influenced by a linear $Q$, so that the spall velocity is also strongly affected. It is therefore useless to attempt to calculate spall velocities in weak decaying shock problems without first replacing the linear $Q$ by some physical model of damping such as the Voigt model. While the Voigt model may not be the best physical model, it is at least free from arbitrary numerical parameters such as zone size and shape. Furthermore, with the Voigt model one can show that a fundamental quantity, the integrated momentum per unit surface area of the pulse, is independent of the viscosity coefficients. Integrating Eq. (41) in the limit of large radius gives 


$$
M \sim \frac{\phi_{0}}{r} \int_{-\infty}^{\infty} \sqrt{\frac{\sigma}{\pi}} e^{-\sigma \tau^{2}} d \tau=\frac{\rho \phi_{0}}{r} .
$$

This is an important quantity because it can be measured experimentally and because it is the impulse that can be delivered by the blast pulse if it is absorbed inelastically. In the case of an underground explosion the surface spall acts as the absorber, carrying most of the momentum of the blast pulse with it as it flies up from the surface. The significance of the result, Eq. (42), is that one can expest to calculate the impulse delivered by the blast wave correctly, independently of the viscosity coefficients. Although it may not be possible to calculate the spall velocity correctly because realistic viscosity coefficients are not known or because they must be made abnormally large for numerical smoothing, it should still be possible to correctly calculate the impulse delivered by the blast wave.

Knowledge of the decay law imposed by by the damping mechanism is also useful. The decay law for the Voigt model obtained from Eq. (41) is

$$
u \sim C_{1}^{-1 / 2} r^{-3 / 2}
$$

This result can be used to infer the decay rate for other types of physical damping mechanisms that may be added to the model. For example, the adiabat and Hugoniot are usually represented by the single curve ( $\mathrm{Eq} .12)$ in calculations because physically they are practically identical for many solids not shocked above the melting point. However, some earth materials exhibit porosity, so that, after shocking, the material folluws a different adiabat lying below the adiabat for the unshocked material. As a result the shock pulse undergoes an additional attenuation in traveraing the material, and the decay law follows a higher inverse power than 1.5. The difference can then be asc ribed to the porosity.

\section{COMPUTATION OF SURFACE WAVES}

The second objective of the numerical analysis work has been to make certain that the Tensor program can accurately calculate Rayleigh wave propagation. This has been accomplished by comparing Tensor numerical solutions with exact mathematical solutions. The impact of spall striking the earth's surface can be approximately represented by a distributed time-varying pressure pulse applied to the surface of a uniform elastic halfspace. Although they do not seem to be noted or referred to as such, Green's Functions for this problem have been determined: exactly, in the twodimensional case (Sherwood, 1958); and asymptotically, in the three-dimensional case (Lamb, 1904).

The two-dimensional Green's Function may be used to compute the response to a pressure applied to an infinite strip with a time-dependence given by

$$
P(t)=\frac{\tau_{0} L}{\pi\left(\tau_{0}^{2}+t^{2}\right)} .
$$

This particular time function is the one used by Lamb in his asymptotic solution for the Rayleigh wave. The integral of this function with respect to time gives 
$L$, the impulse per unit surface area. The parameter $\tau_{0}$ has the units of time and specifies the duration of the pressure pulse, Figure 13 shows a section through the half-space. The spatial distribution is specified by

$$
P=\left\{\begin{array}{ll}
P(t) & -a \leq x \leq a \\
0 & a<|x|
\end{array} .\right.
$$

The half-space is assumed to behave elastically, and its properties are completely specified by the density, bulk modulus, and shear modulus.

The Green's Function for the twodimensional line-source problem is given in terms of the polar coordinates shown in Fig. 13. Let

$$
\begin{aligned}
& \theta_{0}= \begin{cases}\operatorname{arccosh}\left(\frac{\alpha t}{\mathrm{R}}\right), & \alpha \mathrm{t} \geq \mathrm{R} \\
-i \arccos \left(\frac{\alpha \mathrm{t}}{\mathrm{R}}\right), & \alpha \mathrm{t}<\mathrm{R}\end{cases} \\
& \psi_{\mathrm{O}}= \begin{cases}\operatorname{arccosh}\left(\frac{\beta \mathrm{t}}{\mathrm{R}}\right), & \beta \mathrm{t} \geq \mathrm{R} \\
-i \arccos \left(\frac{\beta \mathrm{t}}{\mathrm{R}}\right), & \beta \mathrm{t}<\mathrm{R}\end{cases}
\end{aligned}
$$

$\delta_{R}^{\Delta}=\frac{1}{\pi \rho \beta R}$ Real $\left\{\frac{-i \gamma\left[1-2(\gamma \sin \theta)^{2}\right] \cos \theta \sin \theta \cot \left(-i \theta_{0}\right)}{\left(4(\gamma \sin \theta)^{3}\left[1-(\gamma \sin \theta]^{2}\right\}^{1 / 2} \cos \theta+\left\{1-2(\gamma \sin \theta)^{2}\right\}^{2} \sin \theta\right]}\right\}$

$\delta_{\phi}^{\Delta}=\frac{1}{\pi \rho \beta R} \operatorname{Real}\left\{\frac{-i \gamma\left[1-2(\gamma \sin \theta)^{2}\right] \cos \theta \sin \theta}{\left[4(\gamma \sin \theta)^{3}\left[1-(\gamma \sin \theta)^{2}\right]^{1 / 2} \cos \theta+\left[1-2(\gamma \sin \theta)^{2}\right]^{2} \sin \theta\right]}\right\}$

and

${ }_{\delta}^{\mathrm{W}}=\frac{1}{\pi \rho \beta \mathrm{H}} \operatorname{Real}\left\{\frac{-\mathrm{i}\left[1-\left(\gamma^{-1} \sin \psi\right)^{2}\right]^{1 / 2} \sin \psi \sin 2 \psi}{4(\sin \psi)^{3}\left[1-\left(\gamma^{-1} \sin \psi\right)^{2}\right]^{1 / 2} \cos \psi+\gamma^{-1} \sin \psi(\cos 2 \psi)^{2}}\right\}$

$\delta_{\phi}^{\mathrm{W}}=\frac{1}{\pi \rho \beta K} \operatorname{Real}\left\{\frac{\mathrm{i}\left[1-\left(\gamma^{-1} \sin \psi\right)^{2}\right]^{1 / 2} \sin \psi \sin 2 \psi \cot \left(-\mathrm{i} \psi_{\circ}\right)}{4(\sin \psi)^{3}\left[1-\left(\gamma^{-1} \sin \psi\right)^{2}\right]^{1 / 2} \cos \psi+\gamma^{-1} \sin \psi(\cos 2 \psi)^{2}}\right\}$. 
The branch cut for the square root is chosen so that the real part is always positive:

$$
\sqrt{\rho \mathrm{e}^{\mathrm{i} \Phi}}=\sqrt{\rho} \mathrm{e}^{\mathrm{i} \Phi / 2},-\pi<\Phi \leq \pi
$$

Note that $\delta_{\mathbf{R}}$ and $\delta_{\phi}$ are zero for $\alpha \mathrm{t}<R$. To transform the Green's Function to cartesian coordinates, put

$$
\begin{aligned}
& \phi=\arctan (x / z), \quad R=x^{2}+z^{2} \\
& \delta_{x}=\delta_{R} \sin \phi+\delta_{\phi} \cos \phi \\
& \delta_{z}=\delta_{R} \cos \phi-\delta_{\phi} \sin \phi .
\end{aligned}
$$

To obtain the solution for the specified time function and spatial distribution, one must convolute these functions with the Green's Function. Thus the displacements are given by

$$
\begin{aligned}
\Delta_{z}(x, z, t)= & \frac{1}{2 a} \int_{-a}^{a} d \xi \\
& \times \int_{-\infty}^{\infty} d \tau \frac{\delta_{z}(x-\xi, z, \tau) \tau_{0} L}{\pi\left[\tau_{0}^{2}+(t-\tau)^{2}\right]}
\end{aligned}
$$

and

$$
\begin{aligned}
\Delta_{x}(x, z, t) & =\frac{1}{2 a} \int_{-a}^{a} d \xi \\
& \times \int_{-\infty}^{\infty} d \tau \frac{\delta_{x}(x-\xi, z, \tau) \tau_{0} L}{\pi\left[\tau_{0}^{2}+(t-\tau)^{2}\right]}
\end{aligned}
$$

The Green's Function and the double integrals are evaluated numerically. On the CDC-7600, approximately $1 / 2 ;$ sec running time is needed to calculate $\Delta_{z}$ and $\Delta_{X}$ at a given point in space and time. Values of $\delta_{R}$ and $\delta_{\phi}$ obtained agree with Sherwood's results. To check the timeconvolution part of the solution, the displacements were computed at a very late time and compared with Lamb's twodimensional asymptotic solution for a line source. In this case, the spatial convolution was omitted. Figure 14 shows the comparison of the vertical displacements at the surface. The solid line is the ccnvoluted Green's Function and the small circles are points from Lamb's solution.

Lamb's asymptotic solution for the surface displacements produced by an infinite line source is given by

$$
\Delta_{x}(x, t)=\frac{-L H \tau_{o}}{\mu\left[\tau_{n}^{2}+\left(t-\frac{x}{c}\right)^{2}\right]}
$$

$$
\Delta_{z}(x, t)=\frac{L K\left(t-\frac{x}{c}\right)}{\mu\left[\tau_{0}^{2}+\left(t-\frac{x}{s}\right)^{2}\right]},
$$

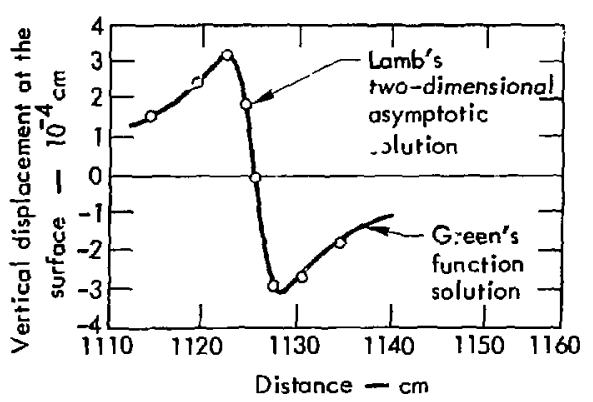

Fig. 14. Comparison of the convoluted Green's function solution (solid curve) with Lamb's two dimensional asymptotic solution (data points). The time is $3.5 \mathrm{msec}$. The sign convention is displacement positive into half space, as shown in Fig. 13. 
where $c$ is the Rayleigh-wave velocity determined by the equation

$$
\begin{aligned}
\mathrm{x}^{3}-8 \mathrm{x}^{2}+\mathrm{x}^{2}\left[24-\left(\frac{\beta}{\alpha}\right)^{2} 16\right] \\
-16\left[1-\left(\frac{\beta}{\alpha}\right)^{2}\right]=0 ; c^{2}=\mathrm{x}^{2},
\end{aligned}
$$

and where

$$
\begin{array}{r}
H=-c^{-1}\left[2 c^{-2}-\beta^{-2}-2\left(c^{-2}-\alpha^{-2}\right)^{1 / 2}\right. \\
\left.\times\left(c^{-2}-\beta^{-2}\right)^{1 / 2}\right] / F \\
K=-\beta^{-2}\left(c^{-2}-\alpha^{-2}\right)^{1 / 2} / F \\
F=B c^{-1}\left[2 c^{-2}-\beta^{-2}\right. \\
\left.-\left(c^{-2}-\alpha^{-2}\right)^{1 / 2}\left(c^{-2}-\beta^{-2}\right)^{1 / 2}\right] \\
-4 c^{-3}\left[\left(\frac{c^{-2}-\alpha^{-2}}{c^{-2}-\beta^{-2}}\right)^{1 / 2}\right. \\
\left.+\left(\frac{c^{-2}-\beta^{-2}}{c^{-2}-\alpha^{-2}}\right)^{1 / 2}\right]
\end{array}
$$

Lamb's solution for the surface displacements at large distances from a point force perpendicular to the surface is given by

$\Delta_{R}(R, t)=\frac{-L H \cos ^{3 / 2} \theta \sin \left(\frac{\pi}{4}-\frac{3}{2} \theta\right)}{2 \mu \sqrt{2 R C} \tau_{0}^{3 / 2}}$

$\Delta_{z}(R, t)=\frac{L K \cos ^{3 / 2} \theta \cos \left(\frac{\pi}{4}-\frac{3}{2} \theta\right)}{2 \mu \sqrt{2 \mathrm{RC}} \tau_{0}^{3 / 2}}$ where

$$
\tan \theta=\left(\mathrm{t}-\frac{\mathrm{R}}{\mathrm{C}}\right) / \tau_{\mathrm{o}}
$$

To make comfarisons, one needs to extend the Lamb point solution to a circular area of radius a by performing a spatial convolution over the surface.

$$
\begin{aligned}
\Delta_{R}^{\prime}(\vec{x}, \tau)= & \frac{1}{\pi a^{2}} \iint_{\text {disk }} \\
& \times \Delta_{R}\left(\left|\vec{x}-\vec{x}^{\prime}\right|, t\right) d \sigma^{\prime} \\
\Delta_{z}^{\prime}(\vec{x}, t)= & \frac{1}{\pi a^{2}} \iint_{\text {disk }} \\
& \Delta_{z}\left(\left|\vec{x}-\vec{x}^{\prime}\right|, t\right) d \sigma
\end{aligned}
$$

For $|\vec{x}-\vec{x}| \gg a_{a}$ these formulae reduce to

$$
\begin{array}{r}
\Delta_{R}^{\prime}(R, t)=\frac{2}{\pi a^{2}} \int_{-a}^{a} \Delta_{R}(R-\xi, t) \\
\quad \times \sqrt{a^{2}-\xi^{2}} d \xi
\end{array}
$$

$$
\begin{array}{r}
\Delta_{z}^{\prime}(\mathrm{R}, \mathrm{t})=\frac{2}{\pi \mathrm{a}^{2}} \int_{-\mathrm{a}}^{a} \Delta_{z}(\mathrm{R}-\xi, \mathrm{t}) \\
\quad \times \sqrt{\mathrm{a}^{2}-\xi^{2}} \mathrm{~d} \xi .
\end{array}
$$

Several properties of Lamb's asymptotic solution are interesting. First we note that the time integral of the source function $P(t)$ is independent of $\tau_{0}$, and equal to the constant L. Furthermore, 


$$
\operatorname{limit}_{\tau \rightarrow 0} P(t)-\left\{\begin{array}{lll}
0 & \text { if } t \neq 0 \\
\infty & \text { if } t=0
\end{array}\right.
$$

Hence,

$\operatorname{limit}_{\tau_{0} \rightarrow 0} \frac{P(t)}{L}=\delta(t) \equiv \underset{\text { the Dirac Delta }}{\text { Function. }}$

since

$\operatorname{limit}_{0} \frac{1}{L} \int_{-\infty}^{\infty} P(t) f(t) d t$

$$
=\int_{-\infty}^{\infty} \delta(t) f(t) d t=f(0)
$$

Therefore, in the limit $\tau_{\mathrm{o}} \rightarrow 0$, Lamb's point source solution gives the asymptotic form of the Green's Function for the Rayleigh wave in three dimensions. As a result one can easily find the Rayleigh wave from any spatial distribution of normal surface forces with arbitrary time variation. To get the time variation, convolve the given source function with the Green's function. To get the contribution from the spatial distribution, perform a double convolution of the Green's Function over the surface area of the source. At large distances from the source, this reduces to a single convolution inter.ral as shown in Fig. 15.

The Rayleigh-wave solutions given are for purely elastic response. The first half of this chapter emphasized that, in the case of the weak decaying shock wave, the numerical methods actually compute the response for a type of Voigt solid. This is also true for the Rayleigh wave. Treatment of the Rayleigh propagation problem by a parallel approach would require the three-dimensional solution

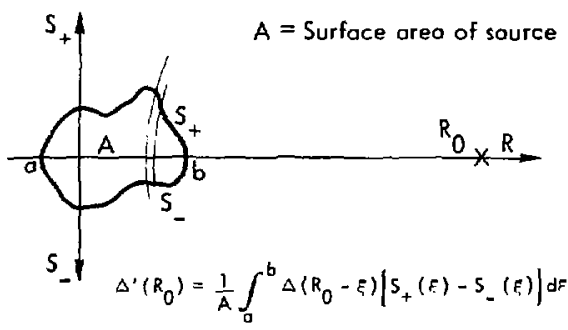

Fig. 15. Reduction of a double spatial convolution to a single integral.

of Lamb's problem for a Voigt solid. Unfortunately, this is a formidable problem in mathematical analysis.

Newlands (1954) attempted to find the Green's function for a normal force (the solution for a force applied to a point on the surface with $\delta$-function timedependence) but did not succeed in getting a closed-form answer. Newlands did, however, show that the Rayleigh pulse is dispersed, with group velocity increasing monotonically with frequency. Newlands also obtained an asymptotic solution for the Green's function in terms of integrals of the form

$\mathscr{L}(\xi)=\int_{0}^{\infty} \sqrt{x} \sin \left(\xi x+\frac{1}{3} x^{3}\right) d x$

$K(\xi)=\int_{0}^{\infty} \sqrt{x} \cos \left(\xi x+\frac{1}{3} x^{3}\right) d x$.

These functions are similar to Airy functions but are not tabulated in standard references. An approximate numerical evaluation of $\mathscr{L}(\xi)$ is plotted in Fig. 16. When $\xi$ is negative, the method of stationary phase may be used to derive the 


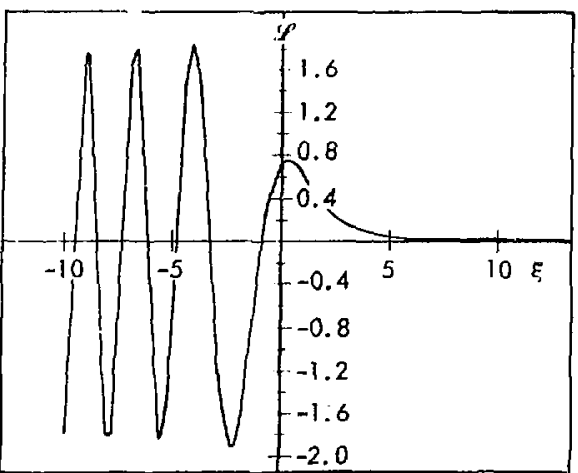

I'jg. 16. Newlands' asymptotic solution for the Rayleigh vertical displacement in a Voigt medium.

asymptotic form also noted in Fig. 16. Newlands' asymptotic solution for the vertical displacement is

$$
\Delta_{\mathrm{z}}(r, t) \sim \frac{A}{\mathrm{r}}[\mathscr{P}(\xi)+K(\xi)]_{1}
$$

where

$$
\xi=(t-r / i)(B r)^{-1 / 3} \text {. }
$$

The constants $A$ and $B$ are functions of the elastic moduli $i$ and the Voigt viscosity coefficients. This solution is accurate only when the absolute value of the retarded time is small in comparison with the travel time. Thus the true solution goes to zero at some large negative value of $\xi$, whereas the approximate result con. tinues on indefinitely at constant amplitude. Figure 16 shows the dispersive character of the pulse, with the high frequencies arriving first and the lowest frequencies last. Newlands' approximate solution also shows that the pulse amplitude is inversely proportional to the first power of the range. In the purely elastic case, the amplitude is inversely proportional to the square root of the range.

Thus the Voigt viscosity affects the cylindrically diverging surface wave and the spherically diverging blast wave in a similar manner, increasing the power of the inverse decay law by one half.

The Rayleigh pulse and blast wave cases are also similar, in that the Voigt viscosity determines the gross features of the asymptotic wave but has negligible effect on them at early times and short range. This is an jmportant observation, particularly as it relates to the convergence of numerical solutions containing linear $Q$ damping terms. In the first half of this chapter it was shown that the traditional linear $Q$ is essentially a Voigt viscosity but with nonphysical viscosity coefficients proportional to the spatial difference interval. It was also demonstrated that the presence of these terms ensures that the late-time pulse amplitude follows a viscous decay law. This poses an apparent paradox. If there are viscosity terms in the traditional linear $Q$ finite-difference programs, then how can the numerical solutions ever converge to the asymptotic solutions for purely elastic response? Obviously, by making the difference interval smaller, one reduces the artificial viscosity coefficients proportionately; but this will not affect the asymptotic decay rate, since it is independent of the magnitude of the viscosity coefficients. No matter how small one makes the difference interval, there is still a finite viscosity term in the equations.

This paradox can be resolved, and the opening statement of the preceding paragraph can be supported, by considering a 
simple example. In one spatial dimension, the planar response of a Voigt solid is governed by Stokes' equation. Eq. (29). The Green's function for a source at the origin is obtained by solving Eq. (29) with a source at the origin having a $\delta$-function time-dependence. The solution for the Green's function is

$G\left(X, t ; 0, t^{\prime}\right)=\frac{A}{\sqrt{v X}} \exp \left[\frac{-\alpha^{3}}{2 \nu X}\left(\tau-\tau^{\prime}\right)^{2}\right]$.

where $A$ is a constant and $\tau$ is the retarded time. The spatial variable $\mathrm{X}$ has bcen used instead of $r$, since planar rather than spherical geometry is being considered. The response for any kind of time-dependence of the source $\psi_{0}\left(\tau^{\prime}\right)$ is then given by convolution with the Green's function:

$$
\begin{aligned}
\psi(\mathrm{X}, \mathrm{t})=\frac{\mathrm{A}}{\sqrt{\nu \mathrm{X}}} \int_{-\infty}^{\infty} \psi_{0}\left(\tau^{\prime}\right) \\
\\
\quad \times \mathrm{e}^{-\beta\left(\tau-\tau^{\prime}\right)^{2}} \mathrm{~d} \tau^{\prime},
\end{aligned}
$$

where

$$
\beta=\alpha^{3} /(2 \nu \mathrm{X})
$$

To make the example as simple as possible, consider a rectangular time pulse of width 2a. Then the solution is

$$
\begin{gathered}
\psi(\mathrm{x}, \mathrm{t})=\frac{\mathrm{A}}{\sqrt{\nu \mathrm{X}}} \int_{\tau-\mathrm{a}}^{\tau+\mathrm{a}} \mathrm{e}^{-\beta \xi^{2}} \mathrm{d \xi} \\
=\frac{\mathrm{A}}{\sqrt{\nu \mathrm{r}^{\beta}}} \int_{(\tau-\mathrm{a}) / \beta}^{(\tau+\mathrm{a}) / \beta} \mathrm{e}^{-\mathrm{y}^{2}} \mathrm{dy} .
\end{gathered}
$$

Then, at the center of the pulse, the amplitude is

$$
\psi_{\xi}=A^{\prime} \int_{-a \sqrt{\beta}}^{2 \sqrt{\beta}} e^{-y^{2}} d y .
$$

For large $\beta$ corresponding to small $\mathrm{x}$, this reduces to

$$
\begin{aligned}
\psi_{\xi}(\mathrm{x})=\mathrm{A}^{\prime \prime} & {\left[1-\frac{1}{\mathrm{a}} \sqrt{\frac{2 \nu}{\pi \alpha^{3}}} \mathrm{e}^{-\frac{\mathrm{e}^{2} \alpha^{3}}{2 \nu \mathrm{x}}}\right.} \\
& \left.\times\left(\sqrt{\mathrm{x}}-\frac{\nu \mathrm{x}^{3 / 2}}{\mathrm{a}^{2} \alpha^{3}}+\ldots\right)\right],
\end{aligned}
$$

and, for small $\beta$ corresponding to large $x$,

$$
\begin{aligned}
& \psi_{\zeta}(x)=A^{1, i}\left[a \sqrt{\frac{\alpha^{5}}{2 \nu \bar{Y}}}\right. \\
& \left.-\frac{a^{3}}{3}\left(\frac{a^{3}}{2 v x}\right)^{3 / 2}+\ldots\right]
\end{aligned}
$$

If the material response were purely elastic, the rectangular pulse would simply maintain its shape anc amplitude. In the visco-elastic case, the pulse maintains its shape and amplitude during the initial stages of travel, except for a very slight rounding at the edges. Then there is a transition stage in which the pulse changes shape and its amplitude begins to decay. Finally the asymptotic state is reached, in which the pulse shape assumea Gaussian form and the amplitude becomes inversely porportiunal to the square root of the range. How these different stages arise is easily understood by referring to the convolution integral, 
Eq. (81). For short ranges, the width of the Gaussian Green's function, Eq. (80), is very narrow in comparison with the width of the rectangular pulse. Convolution with the rectangular pulse simply reproduces the rectangular pulse. When the range is sufficiently great, the width of the Green's function becomes comparable to the width of the initial rectangular source pulse. Convolution then strongly alters the shape and amplitude of the initial pulse. Finally, at very great range, the Gaussian Green's function becomes much wider than the initial rectangular pulse. Convolution of the two functions then simply reproduces the Green's function except for change of amplitude scale.

The viscosity $v$ appears in the solution, Eq. (81), as a scale factor multiplying the range $x$. Thus the effect of reducing the viscosity is to delay the onset of the asymptotic stage. By making the viscosity vanishingly small, the range at which the asymptotic stage begins can be made to approach infinity. This behavior explains why solutions obtained for numerical calculations with the traditional linear $Q$ will converge to elastic solutions in the limit of vanishingly small difference interval. Unfortunately, practical computer calculations must be made with a finite mesh interval, usually large, perhaps no smaller than about $5 \%$ of the width of the purely elastic disturbance. In decaying-wave calculations, the transition to the asymptotic stage occurs well within the ranges of interest, for any practical choice of zoning.

This effect was shown in the blastwave calculations and is also evident in the calculation of the elastic Rayleighwave problems presented in the remain- der of this chapter. Sherwood's (1958) solution is exact at all ranges and times and so offers a good opportunity to observe how the numerical solutions increasingly deviate from the elastic solutions as the range increases. Figure 17 shows a comparison of a tensci. finite-difference solution and the mathematical solution Eq. (58) for the vertical displacement produced by a pressure pulse applied to an infinite strip. The particular choice of units and parameters in this example correspond to the conditions of a physical experiment performed by Sherwood to check his mathematical solution. The density, bulk modulus, and shear modulus in this calculation are $2.77 \mathrm{~g} / \mathrm{cm}^{3}, 4.88 \times 10^{11}$ dyne $/ \mathrm{cm}^{2}$, and $2.74 \times 10^{11}$ dyne $/ \mathrm{cm}^{2}$, respectively. The compressional velocity, $\alpha$, and the shear velocity, $\beta$, appearing in Sherwood's

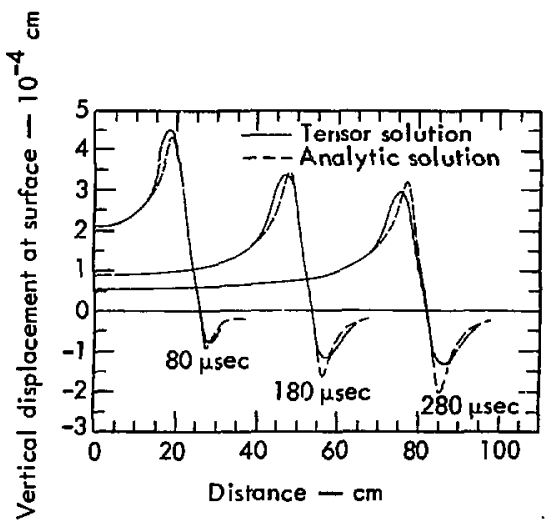

Fig. 17. Comparison of a Tenson finitedifference solution with the analytic solution for the Rayleigh wave produced by a surface load applied to an infinite strip. The sign convention is displacement positive into half spacc, as shown in Fig. 13. 
solution [Eqs. (46) through (57)] are related to the bulk modulus $k$ and the shear modulus $\mu$ by

$$
\begin{aligned}
& \alpha=\left[\left(\mathrm{k}+\frac{4}{3} \mu / \rho\right)\right]^{1 / 2} \\
& \beta=(\mu / \rho)^{1 / 2} .
\end{aligned}
$$

The Voigt bulk and shear viвcosities are both $1.5 \times 10^{5} \mathrm{~g} / \mathrm{cm} / \mathrm{sec}$. The value for the impulse per unit area $L$ in Eq. (44) is (for unit thickness) $2.46 \times 10^{3}$ dyne sec/ $\mathrm{cm}^{2}$. This load is applied uniformly over a strip $8 \mathrm{~cm}$ wide centered at the origin (see Fig. 13). The characteristic time $\tau_{0}$ appearing in Eq. (44) is $5 \times 10^{-6} \mathrm{sec}$.

The representation of the spatial and temporal variation of the applied loading is necessarily approximate in the finitedifference calculations. The degree of approximation used in this comparison test is about the same as that uzed in the simulation experiments of Chapters $\mathrm{V}$ and VI. Figure 18 shows the approximation of Eq. (44) used in the Tensor calculation, along with a plot of $\mathrm{Eq}$. (44) for comparison. The area under the two curves is exactly equal, corresponding to the same impulse per unit area.

The spatial difference interval used is $1 \mathrm{~cm}$ in both the vertical and horizontal directions, so that the applied load is spatially distributed only over eight zones (actually over only four zones in the computations, because symmetry allows one to replace the half plane by a quarter plane with a reflecting boundary along the vertical edgel. Despite this relatively crude modeling of the driving load the agreemert between the numerical and exact mathematical solution is sern to be

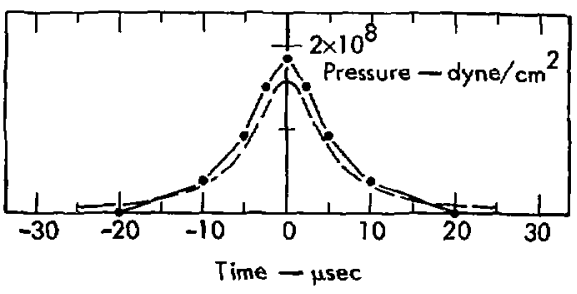

Fig. 18. Source time-dependence for numerical runs, compared with analytical source function. Areas under the two curves are equal.

quite good at the earliest time shown in Fig. 17. The differences between the two solutions can be made indistinguishable at the first time shown, by reducing the spatial difference interval to $0.5 \mathrm{~cm}$ and extending the approximation of the temporal dependence to $\pm 40 \mu \mathrm{sec}$. This degree of resolution in a problem with two spatial dimensions permits calculation of only the first few instants of motion because of the amount of machine time required. Even the more approximate results displayed in Fig. 17 required several hours of CDC 7600 time to compute. The problem is complicated by the presence of the P-wave, which travels at about twice the Rayleigh velocity. The rinite-difference mesh must, of course, terminate at some depth and range. The $P$-wave will reflect from these boundaries, propagate back into the Rayleighwave region, and interfere with it. Thus the mesh must cover a much larger area than just the region where the Rayleighwave solution is to be computed. The total number of mesh points can be reduced somewhat by increasing the difference interval outside the Rayleigh-wave region. 
As can be seen from the results displayed in Fig. 17, the numerical solution begins to deviate from the analytical solusolution as the Rayleigh wave propagates. The amplitude of the numerically calculate $J$ wave is decaying much more rapidly with range. Unfortunately, the approach to the asymptotic state is very slow for the infinite strip source. Given the amount of machine time required, it is not practical to continue the calculation muck beyond three or four hundred microseconds.

If the calcuiations were continued further in time, one would expect the amplitude of the numerical solution to become inversely proportional to the square root of the range in accordance with the results given for a Voigt so:id. The elastic solution, on the other hand, will approach the constant amplitude form shown in Fig. 14.

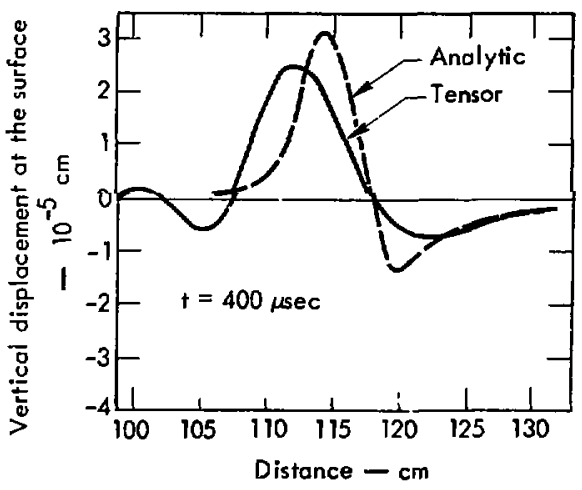

Fig. 19. Comparison of a Tensor finitedifference solution with Lamb's asymptotic solution for the Rayleigh wave produced by a surface load applied to a circular area.
The Rayleigh wave radiated from an impulse applied to a circular area approaches the asymptotic form much more rapidly and is therefore more convenient for studying the asymptotic decay rate of the numerical solution. The solution for the elastic problem is given by Eqs. (66), (67), (71), and (72). Figure 19 shows a comparison between numerical and mathematical solutions to this problem. The elastic constants, impulse time function (Fig. 18), and mesh size are the same as for the infinite strip problem. The impulsive load is distributed uniformly over a circular area of $3-\mathrm{cm}$ radius, so that the total impulse delivered is $6.96 \times 10^{4}$ dyne-sec. The Voigt bulk and shear viscosities have been reduced to $0.75 \times 10^{5} \mathrm{~g} / \mathrm{cm} / \mathrm{sec}$. According to Newlands, the asymptotic Rayleigh displacement amplitude in a Voigt medium should be proportional to the inverse first power of the range, instead of to the inverse one-half power as predicted by the elastic theory. The numerical results in Fig. 19 and all of the finite-difference solutions for Rayleigh waves presented in the following chapters are observed to follow an inverse first-power lay decay, in agreement with the predictions for a Voigt solid.

Newlands ${ }^{1}$ results indicate that there should also be some dispersion of the Rayleigh pulse. Although some dispersion is evident in the numerical solution displayed in Fig. 19, one cannot determine (without an exact mathematical solution for the Voigt medium) whether this is entirely the result of viscoelasticity or whether it is induced by numerical truncation. 
The results discussed in this chapter show that the traditional numerical methods (Maenchen and Sack, 1964; Wilkins, 1964; Wilkins, 1969; Cherry et al., 1971) for solving dynamic problems in solid mechanics produce asymptotic answers that are strongly influenced by the artificial viscosity. While in theory these programs can compute the elastic asymptotic response to an impulsive load, in practice they actually compute the response for a type of Voigt solid with viscosity coefficients depending on numerical parameters. The conclusion drawn from this is that the traditional prescription for determining the viscosity coefficients should be abandoned and replaced by constant input coefficients. This modification has been made in my version of the Tensor program. The modified program will accurately calculate blast waves and Rayleigh waves for the Voigt model of a visco-elastic solid. 


\section{Calculation of Spall}

\section{A. DISCUSSION}

The discussion of the last chapter dealt exclusively with the numerical aspects of the computer program. The emphasis was on determining any discrepancies between the numerical solutions of the dynamical equations of momentum conservation and the true solutions to these equations. The other important aspects of the computer model are the relationships describing the loading and strength properties of the rock. The difficulties here are not with the numerical techniques, but rather with obtaining experimental data on the relationships between pressure and volume and with constructing satisfactory failure models. These models are necessary for accurate calculation in the immediate neighborhood of an explosion and for determining the amplitude constant $\phi_{0}$ in Eqs. (41) and (42). To a great extent, the clevelopment of models has been hindered by difficulties in making experimental measurements. Almost all of the data presently available have been obtained from static experiments (see, for example, Handin et al., 1967; Stephens et al.. 1970b). A new experimental program for making dynamic measurements during shock loading and unloading has been recently initiated by D. B. Larson (Lawrence Livermore Laboratory, personal communication, 1971), but no data have been published yet. Despite their relative simplicity, loading and failure models developed from the static data have in the past proved adequate for predicting nuclear explosion cratering in the earth (Cherry, 1967;
Terhune et al., 1970). Based on this experience it would be reasonable to expect that relatively simple models of loading and failure would be adequate for calculating spall impulse; however, to check this, the spall impulse was calculated for a few specific nuclear explosion tests.

The population of explosions for which there are both accelerometer recordings and material property data is very small. Fairly comprehensive data are available for a few of the very large yields detonated within the continental U.S. and for the Plowshare experiments. The Boxcar and Gasbuggy Events were chosen out of this population for calculation. The burial depths of the two shots lie at the opposite ends of the range of scaled burial depths, so the pair are useful for studying depth effects. Boxcar was fired at $115 \mathrm{~B} \mathrm{~m}$ and Gasbuggy at $1292 \mathrm{~m}$. The explosion energies for Boxcar and Gasbuggy were 1200 and $29 \mathrm{kt}$, respectively (Springer and Kinnaman, 1971). Thus the corresponding scaled burial depths were $109 \mathrm{~m} \mathrm{kt}^{1 / 3}$ and $442 \mathrm{~m} \mathrm{kt}^{1 / 3}$. Surface acceleration, velocity, and displacement data were supplied by Sandia Laboratories, Albuquerque, New Mexico (Perret, personal communication, 1971; Perret, 1969). The diameter of the spallation region was estimated by the method described in Chapter II. The spall diameters for Boxcar and Gasbuggy were approximately 2200 and $2400 \mathrm{~m}$, respectively.

The shot depth and diameter of the spall define the boundaries of the region to be calculated. The computing mesh should be as small as possible. The 
explosive motion is radial and symmetrical about an axis perpendicular to the Earth's surface. Therefore, it is sufficient to calculate the motion within a cone with an apex at the shot point, provided a freeslip rigid-wall boundary condition is used on the sides of the cone. The half-angle of the cone is chosen so that the diameter of the circle formed by the intersection of the cone with the surface is slightly bigger than the diameter of the spall disk. Figure 20 shows a section through the Boxcar mesh. The Gasbuggy mesh is similar, but it has twice as many vertical zones because the scaled burial depth is greater. The calculation of Gasbuggy to the same scaled detail would have required four times as many zones because the scaled burial depth is four times greater. This was a little too expensive, so the Gasbuggy calculations were done with slightly less detail.

During the first few microseconds after the detonation, the outward-moving shock vaporizes a spherical volume of rock. A description of the processes occurring during this time is giver by

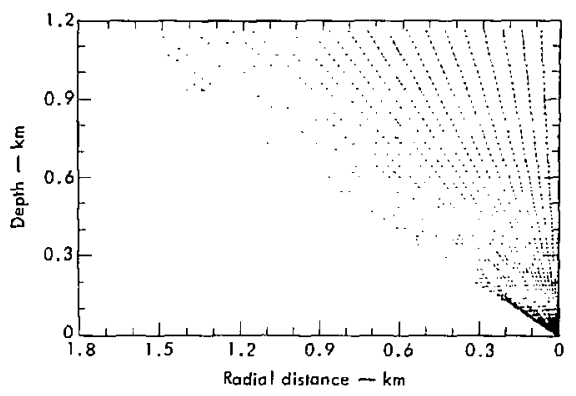

Fig. 20. Lagrangian finite-difference mesh for the Boxcar calculation. The $Z$ axis is a line of axial symmetry.
Rodean (1971). In the case of Boxcar and Gasbuggy, the diameters of the spheres of vaporized rock were approximately 44.0 and $11.0 \mathrm{~m}$, respectively. In the calculations, the driving force of the explosion is supplied by the adiabatic expansion of this gas. Techniques for calculating the volume of vaporized rock and the adiabat have been given by Butkovich (1967, 1971). Figure 21 shows the adiabats for Boxcar and Gasbuggy. The adiabats are calculated for a mixture of $90 \mathrm{wt} \% \mathrm{SiO}_{2}$ plus $10 \mathrm{wt} \% \mathrm{H}_{2} \mathrm{O}$. The pressure and density of the gas are assumed initially uniform throughout the vapor sphere. The initial vapor density is taken to be the density of the surrounding

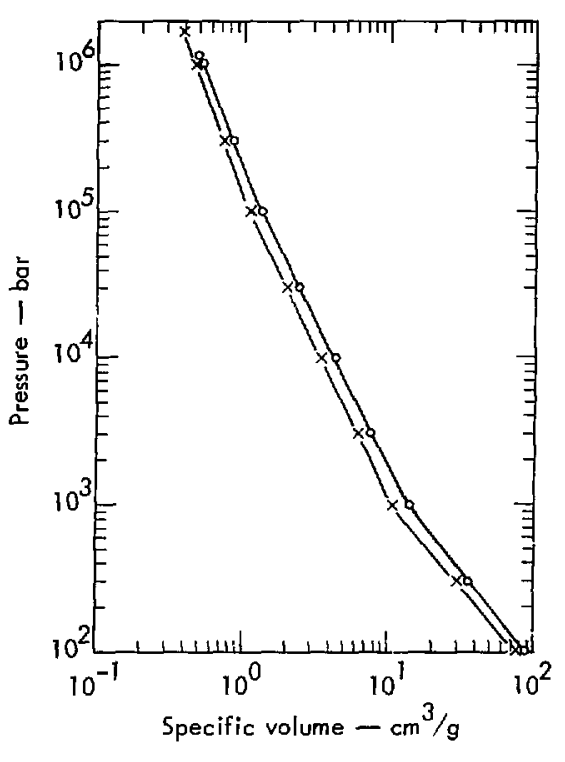

Fig. 21. The vaporized-rock expansion adiabats. The Boxcrir and Gasbuggy curves are indicated by the circles and crosses, respectively. 
rock, and the initial energy density to be the explosive energy release divided by the initial volume of the vapor. The explosion calculations start at the instant the sphere of vaporized rock is formed, so that no more rock is vaporized. Regions initially solid remain solid throughout the calculation. Thus the problem is very similar to the pressurized cavity problem in linear elastic theory, except that the deformation of the cavity wall is now very large because the numerical computations can allow crushing and plastic flow. Instead of being a prescribed time function, the cavity pressure is determined at any instant by the cavity volume and the adiabat. In an actual explosion the density and pressure of the vapor are not uniform; however, if it is not necessary to calculate effects within the cavity, these effects can be ignured.

\section{B. MATHEMATICAL MODELS \\ OF THE MECHANICAL PROPERTIES OF ROCKS}

The response of the rock surrounding the cavity to the pressure load will depend on the mechanical properties of the rock under severe stress. Any model of the mechanical properties of the rock should, at the least, take into account the most important effects observed in the static experiments. Two general types of experiments are performed: 1) loadingunloading tests that measure the pressurevolume response of the rock, and 2) various types of compression, extension and torsion tests that determine the failure characteristics of the rock.

The pressure-volume response is measured by compressing a cylindrical jacketed sample inside a chamber filled with fluid under pressure. The pressurevolume relation is determined by meas uring the change in length and circumference of the sample as a function of the pressure exerted by the piston and fluid. Usually an experiment consists of a complete cycle; the sample is gradually (over a period of an hour or so) compressed till the pressure reaches 30 or $40 \mathrm{kbar}$, then gradually decompressed back to atmospheric pressure. Figure 22 shows the locus of pressure-volume points traced out during an experiment on a sample of rock taken from the bottom of the Boxcar drill hole. Two effects are apparent. First, the pressure-volume relation is nonlinear: as the material is compressed, the bulk modulus steadily increases. Second, the loading and unloading paths are not the same. During loading, some failure and crushing occurs, so the volume of the sample is permanently reduced by the test cycle. The porosity is approximately equal to this volume change divided by the initial

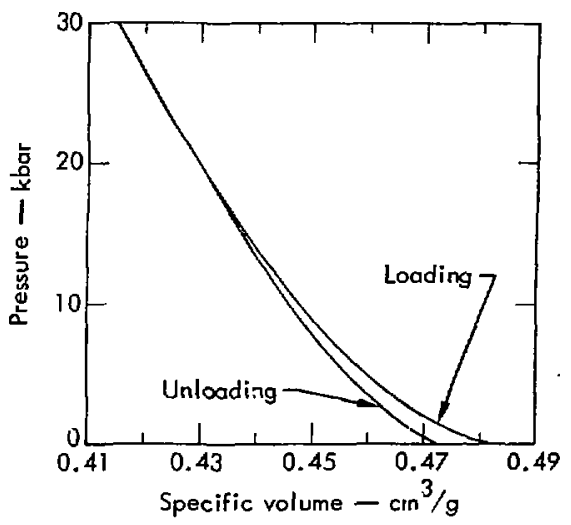

Fig. 22. Experimental pressure-volume relationship for Boxcar rhyolite sample. 
volume of the sample, because a compression to 30 or $40 \mathrm{kbar}$ will squeeze most of the voids out of a sample. It should be emphasized that the data (such as in Fig. 22) obtained from these experiments give the static response of the sample. There is no guarantee that the path traced out in the pressure-volume plane during a dynamic loading cycie of a few millıseconds will be reasonably similar to the path traced out over a period of hours. The loading-unloading path is important because the area enclosed by the path is the amount of mechanical energy irreve sibly converted to heat during the cycle. The pressurevolume path of the rock immediately surrounding the explosion site has a very strong influence on tise net amount of mechanical energy that ultimately gets radiated away. Unfortunately, without any dymanic experimental measurements, one is forced to rely on the da from the static experiments.

The tests for failure in compression, extension and torsion are performed to map a failure surface. The concept of a failure surface is again bazed on static behavior, in that it assumed that failure is determined entirely by the stress state of the material. Since any state of stress is completely specified by three principal stress $r$, it is assumed that there exists a surface in three-dimensional stress space such that the material fails whenever the stress state reaches this surface. The invarients $I_{1}, I_{2 D}^{1 / 2}, I_{3 D}^{1 / 3}$, instead of the stresses, are normally used for the coordinates in plotting the future surface. In terms of the stresses appearing in the dynamic equation of motion [Eqs. (5) through (11)], the invarients are

$$
\begin{aligned}
& I_{1}=3 P \\
& I_{2 D}=\tau_{R} \tau_{Z}+\tau_{R}^{2}+\tau_{Z}^{2}+\tau_{R Z}^{2} \\
& I_{3 D}=\left(\tau_{R}+\tau_{Z}\right)\left(\tau_{R Z}^{2}-\tau_{R}{ }^{\tau} Z\right) .
\end{aligned}
$$

Figure 23 shows several cross sections through the experimentally measured failure surface for Solenhofen limestone. The solid curves are taken from White's (1973) analysis of Handin et al.'s (1967) experimental compression, extension, and torsion data. Unfortunately, SolenLofen limestone is the only earth material for which a sufficient number and type of experiments have been made in order to map out the failure surface. The experimental measurements are difficult to make, especially the torsion tests. Usually, if any failure data at all are available, the only experiment that has been

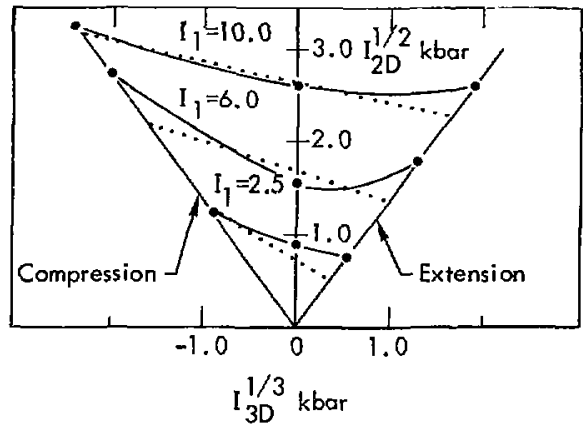

Fig. 23. Experimentally determined failure surface for Solenhofen Limestone (solid lines) after White (1973). The dotted lines show the fit of the simple failure-surface model. For the dotted lines,

and

$$
{ }_{D}^{1 / 2}=10\left[1-\exp \left(\frac{\mathrm{P}}{11}\right)\right]
$$


performed is a triaxial compression test. This test is usually programmed to give the points of intersection of the failure surface with the compression side of the cross section. The triaxial compression is performed by clamping a jacketed cylindrical sample between two pistons. The sample and the ends of the pistons are again inside a chamber filled with lluid. Hydrostatic compression is first performed up to the desired pressure. Then the fluid pressure is fixed and the piston pressure is increased until failure occurs. By making many runs at different confining fluid pressures, the compression branch of the failure surface is measured.

Any physical description of failure should also describe the transient response of the local stresses after failure has occurred. At present there is no satisfactory comprehensive analysis of this problem, even though a great amount of emperical k.lowledge has been accumulated from observations of failures in engineering materials such as metals, glasses, and ceramics. From the aspect of calculating Boxcar and Gasbuggy, a sophisticated theoretical model of failure would not be of much help because the experimenta! data on failure is so limited. In fact, no measurements of failure strength are available for rocks from either the Boxcar or Gasbuggy drill holes. The data that are available are estimated from similar rocks. The lack of data on strength is partly compensated for by the circumstance that strength is not as critical as the pressure-volume behavior in determining the amount of mechanical energy radiated away by the explosion.
My version of the Tensor program uses as simple a model of the mechanical properties as possible. The pressurevolume relatior, Eł. (12), is represented by a polynomial. The coefficients are determined by least-square fitting the polynomial to the tabular experimental data for each rock. The rocks from the Boxcar and Gasbuggy drill holes (see Fig. 22, for example) have smooth, wel.behaved pressure-volume curves, so this procedure works well. The loading path is given by

$$
P=a_{1} x+a_{2} x^{2}+a_{3} x^{3}+a_{4} x^{4}
$$

where

$$
x=\left(V_{0} / V\right)-1
$$

where $V_{0}$ is the uncompressed specific volume and $V$ is the current specific volume.

The unloading path is given by

$$
P= \begin{cases}b_{1}+b_{2} x+b_{3} x^{2} & x<x_{c} \\ 0 & x>x_{c}\end{cases}
$$

The coefficients $b_{1}, b_{2}, b_{3}$ are determined when the maximum compression and pressure are reached. Three simultanenus equations for the three unknowns are obtaineci from restrictions on the unloading curve. The pressure and derivative of the nressure with respect to $x$ should be the same for both loading and unloading curves at the maximum compression. Also, the unloading curve should cross the zero pressure axis at a specified value $x_{c}>0$ corresponding to the crushed volume. These conditions 
will produce an unloading curve similar to the one in Fig. 22.

Ifsually, experimentai data are available for only a 30- or 40-kbar loadingunioading cycle. If the sample were to be be intially loaded to only 10 kbar then the path inllinwed on unloading would be

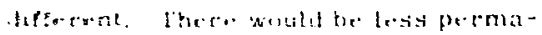

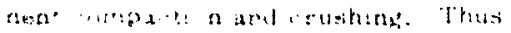

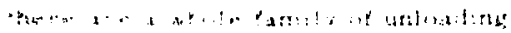

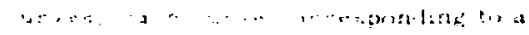

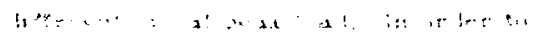

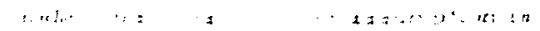

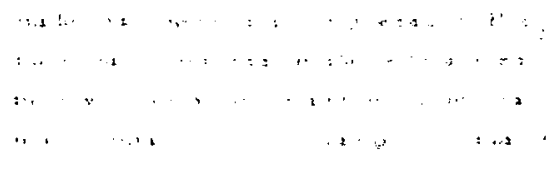
, . , , . , , , , , , , $\therefore$,

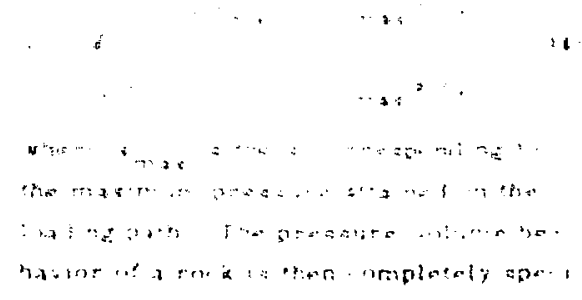

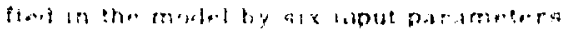
$a_{1} \cdot a_{2}=a_{3} \cdot a_{1} \cdot$ ? and $x_{2}$
The treatment of failure and the description of the failure surface are also as simple as possible. An attempt was made to find a mathematical expression for the failure surface that would have as few parameters as possible, yet still give a gond approximate fit to the available expirimsntal data. The mathernatical expansion for the failure surface jevelopidl by White $(1972)$ allows a clase fitting of any shape of fallure surfare: however, thore to nus much so be sained by using an

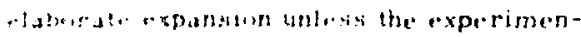

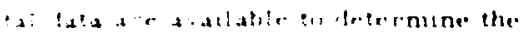

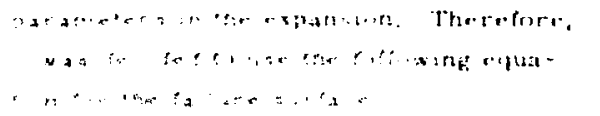

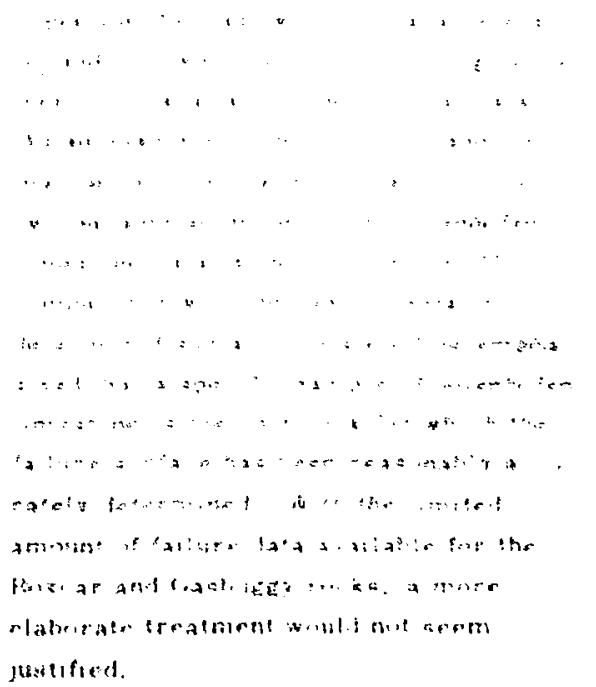


A model of failure must also give a prescription for adjusting the calculated stresses, once $I_{2 D}^{1 / 2}$ penetrates the failure surface. If the material is ductile, the stresses will readjust to maintain $\mathrm{I}_{2 \mathrm{D}}^{1 / 2}$ on the failure surface. If the material is brittle, a crack will open and propagate. and the stress in certain directions will vanish. Except for the case of a simple ductile material, no satisfactory theory of how to adjust the stresses has been developed yet. In this work, the main objective is to calculate the impulse in the blast wave. The mechanical energy radiated away by the explosion is mainly controlled by the pressure-volume behavior and by the limits on the strength of the material. The kind of failure occurring during the early part of the explosion"awily expansion, when the blast wave is bu:ng radiated away, is shear failure in inmpressics. Mnst of the rock close to thr cally will tall ductilely because the

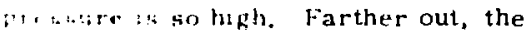
andw : n salure wll be brittle; but, - rn: . the atuisty of the rock to support

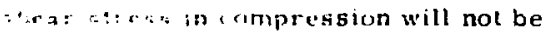
mpiric dontroyed. for example,

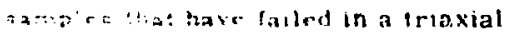
- pracion icat an pall support load lisa : : : : presanal cummunacation).

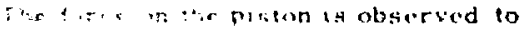

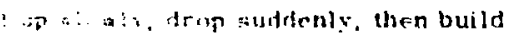
$\Rightarrow$ again, and or nt through many cycles

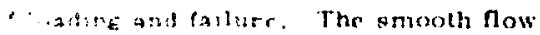

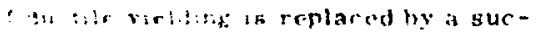
cos:un il sharp. jorky movements, but the abutsty of the ample in support a bran-averagu atrose os maintalned. For Bher reasons, li was decided to reduce the wirewser ' $n \cdot{ }^{\top} Z$, and ${ }^{\top} n Z$ only enough (1) bring $I_{21}^{1 / 2}$ back to the failure surface if it should penetrate it. This is done by reducing each of the stresses by the same proportion.

As long as $\tau_{2 D}^{1 / 2}$ is below the faiiure limit, the stresses are calculated from Eqs. (7) through (9); however, the shear modulus, $\mu$, rather than being taken constant, is calculated from the current bulk modulus, $k$, and a constant-input Pois son's ratio, $\nu$, according to

$$
\mu=\frac{3}{2} \mathrm{k}(1+2 \nu) /(1-\nu),
$$

where

$$
\mathbf{k}=\mathrm{dP} / \mathrm{d} \mathbf{x} .
$$

In addition to a model for the rock properties, the calculations must include the gravitational force, which, in turn, must be balanced by an equilibrium lithostatic pressure distribution. Material at depth is compressed by overburden so that the pressure increase is exponential, but over the relatively shallow shot depth the variation is practically linear. Figure 24 shows the geological cross section for the calculational models of the Boxcar and Gasbuggy sites, and Table 2 gives the properties of each of the rock types in terms of the parameters in the materialproperty model.

The tuff parameters were selected to give the best fit to experimental pressurevolume measurements for TOS -4 tuff (Stephens et al., 1969a). Since no Boxcar tuff samples were actually tested, the TOS-4 tuff data are only an estimate of the pressure-volume behavior for the Boxcar tuff. The rhyolite parameters were selected to fit NTS Vitrophyre from the Boxcar drill hole (U20i) at the $3460-f t$ 


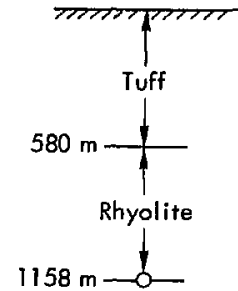

(a)

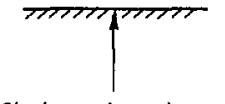

Shale and sandstone mixture

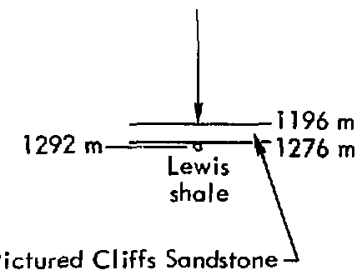

(b)

Fig. 24. Geological cross sections for (a) Boxcar and (b) Gasbuggy Events.

level (Stephens et al., 1970b). The pressure-volume data for Pictured Cliffs sandstone and Lewis shale were also obtained from Stephens et al. (1970b). The density and bulk modulus were obtained from drilling logs (D. Emerson, Lawrence Livermore Laboratory, per- sonal communication. 1971). The properties of the shale-sandstone mixture were estimated from these logs, since wo actual laboratory experiments were made. All of the porosity, Poisson's ratio and the strength parameters were estimated from laboratory measurements of similar rocks (D. R. Stephens, Lawrence Livermore Laboratory, personal communica tion, 1971). The Voigt viscosity coefficients $(\lambda$ and $\eta$ in Table 2 ) were initially selected to damp out zone size-velocity fluctuations. Later these coefficients were increased in order to bring the calculated spall velocity into agreement with the observed.

It should be reemphasized that the experimental data on rock properties and other parameters in the problem are not known with high precision. Even if one had laboratory data on all the properties of a given small chunk of rock, one would still sample only a tiny portion of the

Table 2. Material properties of various types of rosk.

\begin{tabular}{|c|c|c|c|c|c|}
\hline \multirow[b]{2}{*}{ Parameters } & \multicolumn{5}{|c|}{ Rock types } \\
\hline & Tuff & Rhyolite & $\begin{array}{l}\text { Shale and } \\
\text { sandstone } \\
\text { mix }\end{array}$ & $\begin{array}{l}\text { Pictured } \\
\text { cliffs } \\
\text { sandstone }\end{array}$ & $\begin{array}{l}\text { Lewis } \\
\text { shale }\end{array}$ \\
\hline$\rho \mathrm{g} / \mathrm{cm}^{3}$ & 1.80 & 2.08 & 2.25 & 2.50 & 2.63 \\
\hline$a_{1}$ Mbar & 0.05775 & 0.05395 & 0.15 & 0.06067 & 0.1958 \\
\hline$a_{2}$ Mbar & -0.2312 & 1.171 & 0.0 & 1.231 & 3.252 \\
\hline$a_{3}$ Mbar & 1.999 & -2.353 & 0.0 & 7.387 & -1.661 \\
\hline $\mathrm{a}_{4}$ Mbar & -2.951 & $2.4: 9$ & 0.0 & -6.742 & 0.003364 \\
\hline $\mathrm{x}_{1}$ & 0.1374 & 0.02 & 0.0 & 0.02 & 0.0048 \\
\hline $\mathrm{x}_{2}$ & 0.375 & 0.10 & 0.0 & 0.125 & 0.063 \\
\hline Yo Mbar & 0.01 & 0.003 & 0.006 & 0.0025 & 0.0025 \\
\hline$P_{1}$ Mbar & 0.02 & 0.02 & 0.02 & 0.02 & 0.02 \\
\hline$\lambda$ poise & $9.4 \times 10^{7}$ & $9.4 \times 10^{7}$ & $4.7 \times 10^{7}$ & $4.7 \times 10^{7}$ & $4.7 \times 10^{7}$ \\
\hline$\eta$ poise & $4.7 \times 10^{7}$ & $4.7 \times 10^{7}$ & $2.35 \times 10^{7}$ & $2.35 \times 10^{7}$ & $2.35 \times 10^{7}$ \\
\hline Poisson ratio & 0.33 & 0.33 & 0.33 & 0.27 & 0.33 \\
\hline
\end{tabular}


rock surrounding the shot. The measured parameters $P, \nu, a, a_{2}, a_{3}$, and $a_{4}$ in Table 2 represent only the values obtained for a few hundred grams of rock taken from one depth, and the remaining parameters are estimates based on laboratory measurements of similar rocks. Even the shot energy release and burial depth are not precise numbers. The values reported by Springer and Kinnaman (1971) are the best estimates; however, at the time the calculations were done, values were taken from different documents that gave slightly different values. Thus the calculations were actually done with yields differing by a few percent from the values quoted. These uncertainties in the yield make very little difference, because effects scale as the cube root of the yield. The burial depths actually used were 1162 and $1280 \mathrm{~m}$ for Boxcar and Gasbuggy, respectively. The effects of these small differences in burial depth ire insignificant.

\section{COMPARISON OF CALCULA- TIONS WITH EXPERIMENT}

The information obtained from the surface accelerometers allows one to determine the impulse per unit area in the blast wave and the variation of the surface displacement with the range. These are the main things to be calculated.

The calculated blast waven for Boxcar and Gasbuggy are shown in Figs. 25 and 26 as the wave approaches and interacts with the surface. The velocity profiles of the calculated blast waves are in good agreement with the asymptotic form, Eq. (41). Since there are already many uncertainties in the input data for the

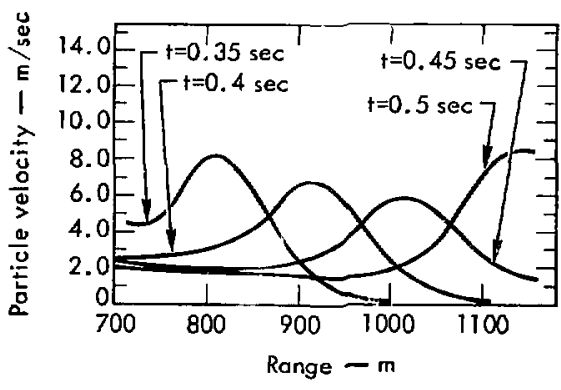

Fig. 25. Calculated vertical variations of particle velocity for Boxcar. Spallation occurs beiween 0.45 and $0.5 \mathrm{sec}$. The surface is at $1162 \mathrm{~m}$.

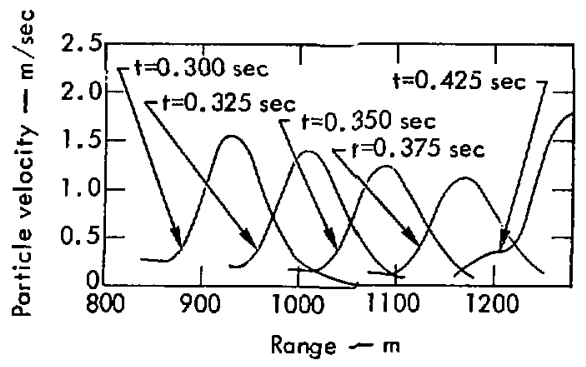

Fig. 20. Caiculation vertical variation of particle velocity for Gasbuggy. Spallation occurs between 0.375 and $0.425 \mathrm{sec}$. The surface is at $1180 \mathrm{~m}$.

calculations, it was felt that especially high accuracy was not required in determining the impulse in the waves. Therefore, instead of performing a machine integration of the momentum of each mesh point within the wave, it was decided to simpiy calculate the vertical moinentum from a radial integration of the asymptotic solutior., Eq. (41), with parameters $\sigma$ and $\phi_{0}$ chosen to fit the 
asymptotic solution to the numerically calculated solution. The calculated impulse per unit area of wave front as the pulse hits the surface is $1.9 \times 10^{7}$ dyne$\mathrm{sec} / \mathrm{cm}^{2}$ for Boxcar and $2.15 \times 10^{6}$ dyne$\mathrm{sec} / \mathrm{cm}^{2}$ for Gasbuggy. These values are for the portion of the wave traveling vertically, corresponding to the profiles in Figs, $2 \overline{5}$ and 26 . The experimental value for Boxcar is obtained by multiplying the effective spall thickness (obtained from Fig. 4) times the density (obtained from the tuff data in Table 2) times the spall velocity (obtained from Fig. 9). It is $4.46 \times 10^{7}$ dyne-sec $/ \mathrm{cm}^{2}$. The methods of analysis in Chapter. II may also be applied to the Gasbuggy accelerometer records, and yield an impulse per unit area of $3.36 \times 10^{6}$ dyne-sec $/ \mathrm{cm}^{2}$. The numerically calculated impulses are 43 and $64 \%$ of the experimental estimates for Boxcar and Casbuggy, respectively.

In Chapter III it was shown that the momentum or impulse of the calculated wave is independent of the Voigt viscosity coefficients. Therefore, to change the calculated pulse momentum, it is necessary to change either the pressure volume relation or the failuie surface. It has been emphasized that there is considerable uncertainty in the material property data: that much of it is, of ecessity, based on estimates from simar rocks. Therefore, attempts were alade to see if the calculated momentum ould be brought $\mathrm{i}$, better agreement with experiment with reasonable changes in the material properties. The Boxcar site is relatively close to other explosion sites where material properiy measureunents have been made, whereas the Casbuggy $\mathrm{sl}^{+} \mathrm{e}$ is igolated. Thus, in the case of Boxcar, one has other data to compare with, to determine what a reasonable material property change might be. For example. Stephens et al. (1969b) have given a compilation of pressure-volume relationships for over 30 Nevada Test Site tuffs and rhyolites. Almost all of the tuffs and rhyolites are identical in chemical composition (I. Borg, personal communication), yet they show great differences in the $P(x)$ relationship. These differences are primarily a function of the porosity and the amount of water in the rock. Trapped water can drasti ally alter the $P(x)$ curve of a porous tuff, eliminating much of the crushability and therefore permitting more of the exfiasive energy to be radiated as mechanical energy. Water also acts si ungly to reduce the shear strength of the rock. The available information on moisture content at points along the Boxcar drill hole indicated that the Boxcar rocks were fairly thoroughly saturated; however, there were no moisture measurements above a depth of $450 \mathrm{~m}$. The single sample that was actually measured (see Fig. 22) is of rhyolite taken from a point near the bottom of the Boxcar sill hole, so that it is necessary to guess at the rock properties farther up the hole. The use of a dry tuff above $580 \mathrm{~m}$ may not have been a good guess; this material is much softer and more crushable than the rhyolite. Furthermore, the Roxcar rhyolite measurement was made on a dry sample. The accur:te measurement of saturated samples is dificult because the sample begins to dry out as soon as it is removed from the drill hole. To make measurements, the samples have to be artificially resaturated. In this case, the records indicate 
that the pressure-volume measurements were made on dry samples. Without the water in the pores, the samples will be softer and more crushable than the same material at depth. There is also a possibility that the strength of the sample could have been greatly overestimated. It is very difficuit to say what a reasonable strength would be for the Boxcar sample, because strength is so extremely sensitive to water content. For example, the strength measurements by H.C. Heard and H. R. Washington (Lawrence Livermore Laboratory, personal cummuniration, 1968) on samples of tuffs and rhyolites taken from nearby drill holes show strengths ranging from 0.08 to 3.0 kbar (at a pressure of 3.5 kbar), depending on saturation. The values of $Y_{0}$ and $P_{1}$ in Table 2 correspond to a strength of about $0.5 \mathrm{kbar}$ at the $3.5-\mathrm{kbar}$ pressure level. Thus, it is possible that the Boxcar rocks were much weaker than assumed in the model. In order to estimate the effect of these uncertainties, it was decided to rerun the Boxcar calculation with greatly reduced porosity and strength and with the tuff replaced by the rhyolite. The failure limit $Y_{0}$ was redured to $100 \mathrm{kbar}$, and $x_{1}$ and $x_{2}$ set to

1. The effect of these changes is to inc rease the impulse to $3.5 \times 10^{7}$ dynesec/cri ${ }^{2}$, which is about $80 \%$ of the experimental value. From this, one is led to conclude that the numerical calculations and the method of obtaining the momentum from the accelerometer records produce reasonably consistent answers. With the kinds of data available, one can hope to get values $f \mathrm{Cr}^{-}$the spall impulse accurate to within about $\pm 25 \%$.
In addition to the spall impulse, it was hoped it would be possible to calculate the shape of the surface displacement and

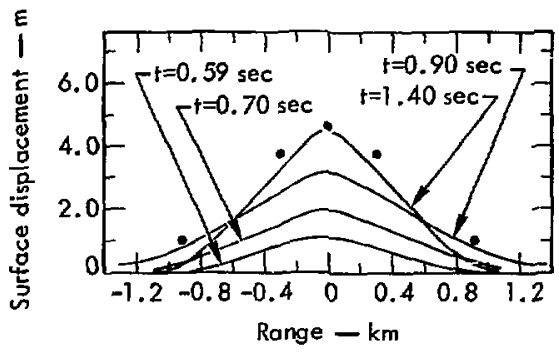

Fig. 27. Calculated Boxcar vertical surface displacement, compared with experimental observations. At $1.4 \mathrm{sec}$, the calculated surface displacement has reached its maximum and the spalled material is beginning to fall back to the Earth. The solid dots are the experimental observations of peak vertical displacement.

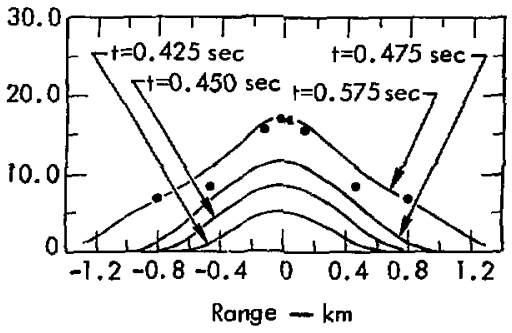

Fig. 28. Calculated Gasbuggy vertical surface displacement, compared with experimental observations. At $0.575 \mathrm{sec}$, the calculated surface displacement has reached its maximum and the spalled material is beginning to fall back to the Earth. The solid dots are the experimental observations of peak vertical displacement. 
the spall radius. The results in Chapter III showed that the spall velocity is strongly dependent on viscosity. Therefore, one cannot expect to calculate spall velocity, unless, of course, the voigt model happens to be a good model for the: medium, and the physical viscosity coefficients are known. Because of this, the viscosity coefficients were adjusted to make the calculated spall velocity agree with the measured value at ground zero. Figures 27 and 28 show the numerically calculated surface displacernent profiles obtained by following this procedure. The experimental maximum observed displacements are also plotted in Figs. 27 and 28 for comparison. The significance of these comparisons is that they show that the shape of the peak displacement profile (and therefore the spall radius) obtained from the caiculations is in good agreement with the experimentally observed shape. It should also be noted that these shapes confirm the assimpticns that were made in calculating the total impulse by Eq. (3). 


\section{Rayleigh-Wave Generation by Spall Impect}

\section{A. PREDICTIONS OF IDEALIZED MODEL COMPARED WITH EXPERIMENT}

The hypothesis to be tested supposes that all, or a significant portion, of the observed surface wave originates from the impact of the free-falling spall plate. An idealized model for calculating the Rayleigh wave to be expected from a surface mpact is that of a uniform elastic half space subjected to an impalse.

Lamb (1904) originally solved this problem for a point force perpendicular to the surface. With suitable generalization, his solution can be used to calculate approximate surface wave amplitudes for the impulse delivered by the spall impact. These amplitudes can then be compared with the experimentally observed surface wave amplitudes to determine if the spall impulse is sufficient to account for the observed amplitudes.

Lamb's solution has already been discussed in Chapter III and is given by Eqs. (66) and (67). It can very easily be extended to include attenuation. In frequency space, the effect of attenuation is to multiply the solution by the damping factor:

$$
D^{(\omega)}=\exp \left[-|\leqslant| r(2 c Q)^{-1}\right] .
$$

The time-dependence $c f$ the source is also included in frequency space by multiplying by the transform of the source function. It happens that the Fourier transform of Lamb's force funciion, Eq. (44), is (for a unit impulse)

$$
\tilde{F}(\omega)=\exp \left(-|\omega| \tau_{0}\right)
$$

Therefore, attenuation can be included by making the substitution

$$
\tau_{0}^{\prime}=\tau_{0}+r(2 c Q)^{-1}
$$

The characteristic time-duration of the spall impact is about twice the spall thickness divided by the compression velocity. This is the time that passes before the pressure developed by the impact can be relieved by a rarefaction from the top of the spall plate. This time interval is approximately $0.2 \mathrm{sec}$ for a 1-Mt explosion. Assuming $Q$ is roughly 150.0 (Knopoff, 1964), one finds that the characteristic attenuation time is comparable to the source time constant at ranges of $\sim 100 \mathrm{~km}$.

The spatial distribution of the spall impulse consistent with the conlie-hat surface displacement profile, id issed in Chapters II and IV, is

$$
I_{a}=\rho d_{0}\left(2 g h_{o}\right)^{1 / 2}\left(1-r / r_{o}\right) .
$$

Lamb's solution, however, applies for all of the im pulse concentrated at a single point. His solution is, for unit impulse, the asymptotic Green's function for the normal force problem. One can get the solution for the distributed impulse by convolving the Green's function solution with the distribution $I_{a}$. The solution requires a double integration over the spall area. However, when the range is large compared to the spall radius, one can approximate the double integration by a single integration with a suitable weight function. To find the appropriate weight 
function, Eq. (3) can be written in terms of rectangular coordinates:

$$
\begin{aligned}
I=2 \rho_{o} d_{o}\left(2 g h_{o}\right)^{1 / 2} \int_{-r_{o}}^{r_{o}} \\
\quad \times \int_{0}^{r_{o}\left[1-\left(x / r_{o}\right)^{2}\right]^{1 / 2}} \\
\times\left[1-r_{o}^{-1}\left(x^{2}+y^{2}\right)^{1 / 2}\right] d y d x
\end{aligned}
$$

On performing the $y$ integration and dividing by $l$, one obtains

$$
1=\int_{-r_{0}}^{r_{0}} \rho_{w} d x
$$

where

$$
\begin{gathered}
\rho_{\mathrm{w}}=3\left(\pi \mathrm{r}_{\mathrm{o}}\right)^{-1}\left[1-\left(\mathrm{x} / \mathrm{r}_{\mathrm{o}}\right)^{2}\right]^{1 / 2} \\
-3 \mathrm{x}^{3} \pi^{-1} \mathrm{r}_{\mathrm{o}}^{-3} \\
x \ln \left(\frac{\mathrm{r}_{\mathrm{o}}}{|\mathrm{x}|}\left\{1+\left[1-\left(\mathrm{x} / \mathrm{r}_{\mathrm{o}}\right)^{2}\right]^{1 / 2}\right\}\right) .
\end{gathered}
$$

The weight function $\rho_{\mathrm{w}} d x$ represents the fraction of the total impulse contained in a strip of surface of width $d x$. A receiver far out on the $x$ axis sees a signal that is the sum of the signals from each one of these strips delayed to the appropriate retarded time. The stipulation that the distance to the receiver is large

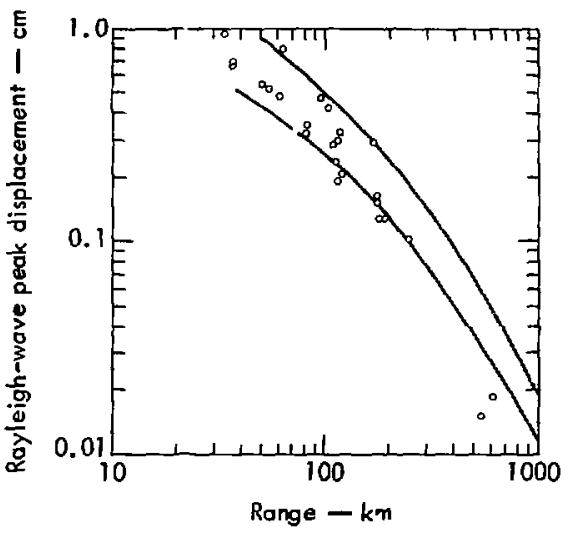

Fig. 29. Comparison of range variation of experimental peak Rayleigh vertical-displacement data with peak amplitudes calculated from the spall impulse. The data are scaled (amplitude proportional to yield) to $1 \mathrm{Mt}$. Events represented by the small circles are Boxcar, Greeley, and halfbeak. The upper spall curve is based on propagation velocities characteristic of the shot point. The lower spall curve is based on propagation velocities characteristic of measured travel times.

compared to $r_{0}$ allows one to make the assumption that signals arriving from different points along a strip have essentially the same delay time. Therefore, the displacements for the distributed source are given by

$$
\Delta \mathrm{R}(\mathrm{r}, \mathrm{t})=\int_{-\mathrm{r}_{\mathrm{o}}}^{\mathrm{r}_{\mathrm{o}}} \rho_{w}(\mathrm{x}) \delta \mathrm{R}(\mathrm{r}-\mathrm{x}, \mathrm{t}) \mathrm{d} \mathrm{x}
$$

Table 3. Parameters for curves shown in Fig. 29.

\begin{tabular}{lllllllll}
\hline Case & $\begin{array}{c}\alpha, \\
\mathrm{km} / \mathrm{sec}\end{array}$ & $\begin{array}{c}\beta, \\
\mathrm{km} / \mathrm{sec}\end{array}$ & $\begin{array}{c}\mathrm{c} / \mathrm{km} / \mathrm{sec} \\
\mathrm{g} / \mathrm{cm}^{3}\end{array}$ & $\begin{array}{c}\mu_{j} \\
\mathrm{dyne} / \mathrm{cm}^{2}\end{array}$ & $\begin{array}{c}\sigma_{0} \\
\mathrm{sec}\end{array}$ & $Q$ & $\begin{array}{c}\mathrm{I} \\
\mathrm{dyne}-\mathrm{sec}\end{array}$ \\
\hline 1 & 2.3 & 1.15 & 1.073 & 2.0 & $2.645 \times 10^{10}$ & 0.2 & 150 & $4.6 \times 10^{17}$ \\
2 & 6.0 & 2.1 & 2.0 & 2.0 & $6.0 \times 10^{10}$ & 0.2 & 150 & $4.6 \times 10^{17}$ \\
\hline
\end{tabular}


and

$\Delta Z(r, t)=\int_{-r_{0}}^{r} \rho_{w}(x) \delta Z(r-x, t) d x$.

These integrals were evaluated numerically for diffement values of $r$ to obtain curves of peak surface vertical displacement versus range. The two curves plotted in Fig. 29 were computed for a 1 -Mt explosion with impulse given by Eq. (4). The parameters for the two cases are given in Table 3 . Case 1 is the upper curve in Fig. 29 and corresponds to material propertîes characteristic of tuff taken from explosion drill holes. Case 2 is the lower curve in Fig. 29 and corresponds to material properties consistent with seismic travel-time measurements of explosion surface waves at ranges of 100 to $200 \mathrm{~km}$. The mathematical model is highly simplified and does not allow spatial variation of surface elevation or geological properties and, therefore, does not take into account scattering and dispersion. However, it should provide a rough estimate of the peak Rayleigh displacements consistent with the observed spall impulse.

Figure 29 also shows the observed vertical Rayleigh displacements for some of the expiosions the spall estimates are based upon. These data were supplied by Environmental Research Corporation, Alexandria, Virginia. The displacemeni amplitudes have all been scaled to $1 \mathrm{Mt}$ by dividing the observed amplitude by the explosion energy in megatons. The periods corresponding to the peak displacements vary from 2 to $5 \mathrm{sec}$, the shorter periods occurring at short range and the longer periods at long range.
The velocities vary between 2.0 and $2.5 \mathrm{~km} / \mathrm{sec}$, which is consistent for Rayleigh waves in the observed period range. The experimental data fall in about the same range as the theoretical estimates. Based on this comparison, one is led to conclude that the estimated spall momentum is sufficient to account for the observed surface wave amplitudes.

\section{B. PREDICTIONS OF COMPUTER MODELS}

The hypothesized spall mechanism of surface wave generation has also been tested by means of computer simulation experiments. Two computer runs simulated two identical explosions in the same material with but one difference. The first run allowed material to fail in tension, permitting spall, while in the second run the material was constrained to behave elastically, thus preventing spall. The effect of spallation could then be demonstrated by comparing the surface waves produced in the two experiments. The simulated experinents were computed by the program and methods described in Chapters III and IV.

Since the amount of detail one can expect to resolve in a calculation depends on the difference interval, the accuracy is necessarily limited by the amount of computing time and memory available. In the simulated-explosion experiments it was necessary to model the explosion neighborhood reasonably well yet also to be able to calculate the surface wave to a distance of 15 or $20 \mathrm{~km}$. This distance is required because the Rayleigh wave is not well-defined or well-separated from other waves at shorter ranges. The calculation of every detail of the explosion 
process starting from a small sphere of vaporized rock would require zone sizes of the order of meters and integration time steps of the order of milliseconds. it would be impractical to calculate the Rayleigh wave to $15 \mathrm{~km}$ with this level of detail. Instead, a minimum zone size of $200 \mathrm{~m}$ is used, and the explosion is modeled by placing an initial compression into the nine zones surrounding the detonation point. The cumputational mesh is reproduced in Fig. 30, where the initially compressed nine zones are indicated by shaded zones. This zoning and representation of the source is the compromise necessary to permit the inclusion of a long propagation path for the Rayleigh wave. In order to test what effect usir: only nine zones to represent the initially compressed material might have, the solutions were recomputed with the mesh interval halved. Mesh refinement cannot be carried very far without drastically reducing the dimensions of the region covered, since halving the mesh interval produces an eight-fold increase in the

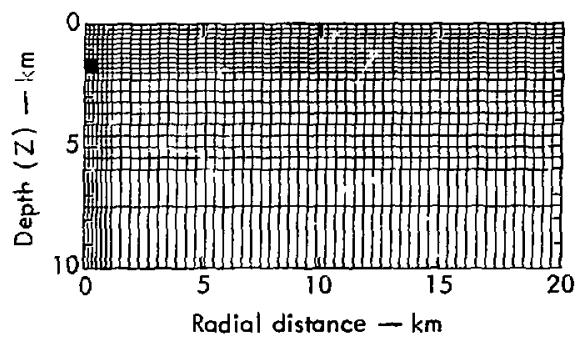

Fig. 30. Finite difference mesh used in the computer simulation experiments. The shaded zones initially contained 1.2 Mt of strain energy of compression. The $\mathbf{Z}$ axis is the centerline of axial symmetry. computing time. The finer-mesh calculation wds run out to a model time oi only $5 \mathrm{sec}$ because of this. The form, shape, and arrival times of the solutions were the same; the only apparent effect was that the solutions differed slightly from point to point. The ratio of the solution obtained on the refined mesh to the solution obtained on the mesh shown in Fig. 30 was $1.00 \pm 0.05$. Thus the error introduced by using only nine zones instead of 36 to represent the initially compressed material is only about $\mathbf{+ 5 \%}$. Since the object of this study is the comparison of relative effects between calculations done on identical meshes with identical parameters (except for iallure). it is felt that the mesh shown in Fig. 30 is adequate.

The initial elastic strain energy within the compressed region is given approximately by

$$
E=(1 / 2)_{\kappa} V_{o}(\eta-1)^{2},
$$

wl. 2 re $\kappa$ is the bulk modulus, $V_{o}$ is the volume, and $\eta$ is the compression. An initial compression of 1.046011 is used. This corresponds to a total strain energy of $1.2 \mathrm{Mt}$. Since the energy density is low, more of this energy is available for elastic radiation than would be the case for an actual explosion with the same total energy release. On the basis of the spall relocity computed, this source is estimated to be roughly equivalent to a yield of between 6 to $9 \mathrm{Mt}$ at a depth of $1700 \mathrm{~m}$.

The variable zoning evident in Fig. 30 permits an inexpensive method of ai $\mathrm{Aw}_{\mathrm{i}}$ ing room for the compression wave to travel outward, thus avoiding the problem 
of interference from reflections at the bottom of the mesh. The vertical zoning extends $b$.yond the level shown to a depth of $15 \mathrm{~km}$.

The equation of state for the model earth in both runs was fairly simple. The pressure was given by

$$
P=\kappa(\eta-1)
$$

for the no-sp :1. case, and by

$$
P= \begin{cases}k(\eta-1) & \eta>1 \\ 0 & \eta \leq 1\end{cases}
$$

for the spall case. The bulk modulus, $k$, was chosen to be $70 \mathrm{kbar}$, the density was $2.0 \mathrm{~g} / \mathrm{cm}^{3}$, and Poisson's ratio was fixed at $1 / 3$. In these experiments the Voigt bui. and shear visccsity coefficients were chosen to be $1.25 \times 10^{9}$ and $4.6875 \times 10^{8}$ poise, respectively. The magnitudes of these viscosities were selected for pureiy numerical reasons. Their purpose is to damp out the short-wave-length noise that develops in finite amplitude sound-wave calculations. The viscosity acts to diffuse momentum over a distance $\Delta x$, which may be estimated from

$\Delta \mathrm{x}=(2 \nu \mathrm{t})^{1 / 2}, \nu=\left(\lambda_{1}+\frac{4}{3} \lambda_{2}\right) \rho^{-1}$,

where $\lambda_{1}$ is the bulk viscosity and $\lambda_{2}$ is the shear viscosity. With the specific elastic constants, the Rayleigh wave takes approximately $15 \mathrm{sec}$ to propagate out to $15 \mathrm{~km}$. This gives a diffusion length of roughly $1.2 \mathrm{~km}$. Therefore, the viscosity will act mainly to damp out short-period noise; however, it should be kept in mind that the computed Rayleigh waves are for a Voigt solid rather than for a purely elastic medium.

Since both runs included gravitation, it was necessary to start calculations with an iritial equilibrium lithostatic pressure distribution within the half space. With $z$ defined positive into the model earth, the lithostatic pressure is

$$
F=\kappa\left[\exp \left(\rho_{0} g z / k\right)-1\right]
$$

The exponential reflects the compression produced by overburden.

The only difference between the two explosion simulations involved the treatment of failure. In the no-spall calculation, no limits were piaced on the calculated stress field. Except for the Voigt viscosity, the material behavior was completely elastic. In the spall calcuiation, however, the failure scheme described in Chapter IV was used. In this particular case, the parameters $Y_{0}$ and $P_{1}$ in Eq. (95) were chosen to be 10 and 20 kbar, respectively. Thus the material had a maximum shear strength of 10 kbar under very high pressure and essentially no strength at zero pressure. The elimination of the ability to support negative pressures and the vanishing of the shear strength at low pressure make spallation possible.

The Rayleigh waves observed in the simulated experiments are displayed in Fig. 31, where the vertical particle velocity is plotted against the range at a time of $15 \mathrm{sec}$. The sign convention has been reversed from that in Fig. 30, so that a positive velocity corresponds to motion directed outward from the model earth. It is unlikely that the Rayleigh waves shown in Fig. 31 are compromised 


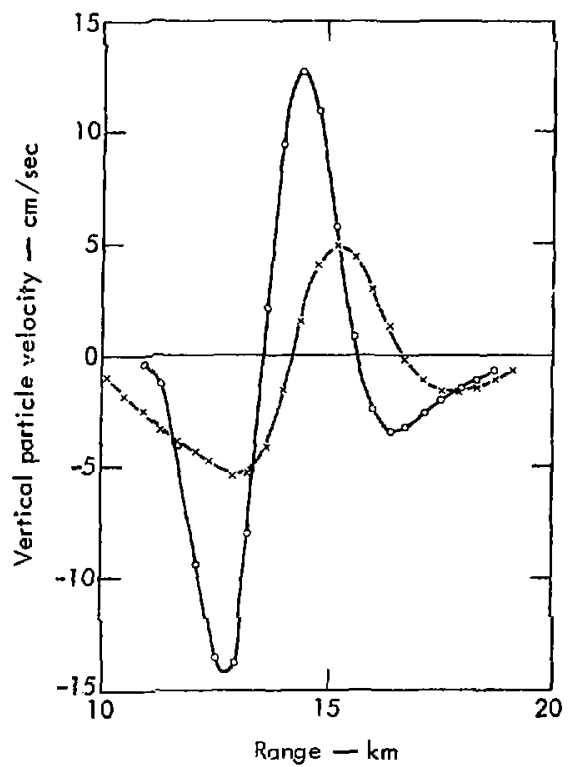

Fig. 31. Comparison of Rayleigh waves calculated in the simulation experiments. Particle velocity is plotted against range. The curve indicated by the small circles is the spallation run. The curve indicated by the small crosses is the elastic run. The time is 15.0 sec.

by interference from either compression waves or sinear waves. With the given elastic constants, the compressional velocity is $2.29 \mathrm{~km} / \mathrm{s} s$, the shear velocity is $1.15 \mathrm{~km} / \mathrm{sec}$, and the Rayleigh velocity is $1.07 \mathrm{~km} / \mathrm{sec}$. Thus any compressional disturbances are located at a range of spproximately $30 \mathrm{~km}$ at a time of $15 \mathrm{sec}$. The shear wave, on the other hand, is only about $1 \mathrm{~km}$ ahead of the Rayleigh wave at this time. The shear wave has a higher decay rate, however, because it diverges spherically. It is unlikely that there is any significant interference from shear motion at $15 \mathrm{~km}$ range.

Comparison of the two runs shows, first, that the surface wave amplitude for the inelastic case is 2.7 times the amplitude for the elastic case and, second, that the wave from the inelastic source is delayed relative to the elastic source. The Rayleigh velocity calculated from elastic theory is $1.07 \mathrm{~km} / \mathrm{sec}$. Thus a delay of about $2.4 \mathrm{sec}$, corresponding to the maximum spall free-fall time, is equivalent to approximately a $2.5-\mathrm{km}$ offset in Fig. 31. In the figure, the first negative peak is offset by about $1.5 \mathrm{~km}$, the fîrst positive peak by about $1.0 \mathrm{~km}$, and the final negative peak by less than $0.5 \mathrm{~km}$.

Exrmination of the computer results in the source region shows that the ground zero surface displacement is much larger in the inelastic case: $12.0 \mathrm{~m}$, compared with $1.9 \mathrm{~m}$ for the elastic case. In the inelastic run, the material in the expected spall region is observed to move in a ballistic trajectory; and the gross features of the spall are the same as those observed in the detailed numerical calculatione described in Chapter IV. The simple theory deseribed by Egs. \{102\}(107) does not take all these features into account, but it does appear to preserve the gross features of the spallation process. The distribution of vertical momentum assumed in the simple model appears to be realistic and the time behavior assumed for the impact appears to be a reasonable approximation. The more complicated behavior of the actual process must, however, account for the fact that the initial phases of both the inelastic and elastic runs have the same sign. If 
the Rayleigh wave could be predicted ex-

stly by the simple spall theory, then on would expect that the initial phase uould have an opposite sign from the elastic case. It is possible that the Rayleigh wave is generated partly by the spall and partly by the traditional elastic process. Since the spall-generated Rayleigh wave is delayed with respect to the elastically generated wave, this could account for the initial phase observed in the spall run.

As a simple experiment, the theoreticai asymptotic solutions for the Rayleigh waves generated by $z$ buried point source and a surface point force were computed. The solu'ion for the buried point source with step-function time-depencence for the displacement potential (corresponding to an explosion) is given by Cagniard

(1962). The solution for the point surface force is just Eq. (67). The displacement potential and the total inpulse were chosen to be $3 \times 10^{12} \mathrm{~cm}^{3}$ and 6.6 $\times 10^{18}$ dyne-sec, respectively. These values correspond to the 6 - to $9 \mathrm{Mt}$ explosion simulated in the numerical experiments. The elastic constants were also the same. Figure 32 shows the resulting variation of the vertical velocity with range, at $15 \mathrm{sec}$, for each source and for a superposition of the two with appropriate time delay. The separate solutions show that the initial motion is downwards for the buried explosive source and upwards for the surface force, although weakly in comparison. The solution for the surface force has been computed with a time delay of $2.5 \mathrm{sec}$, to correspond to the delayed impact of the spall material. The superposition of the two signals with this delay produces a signal similar to
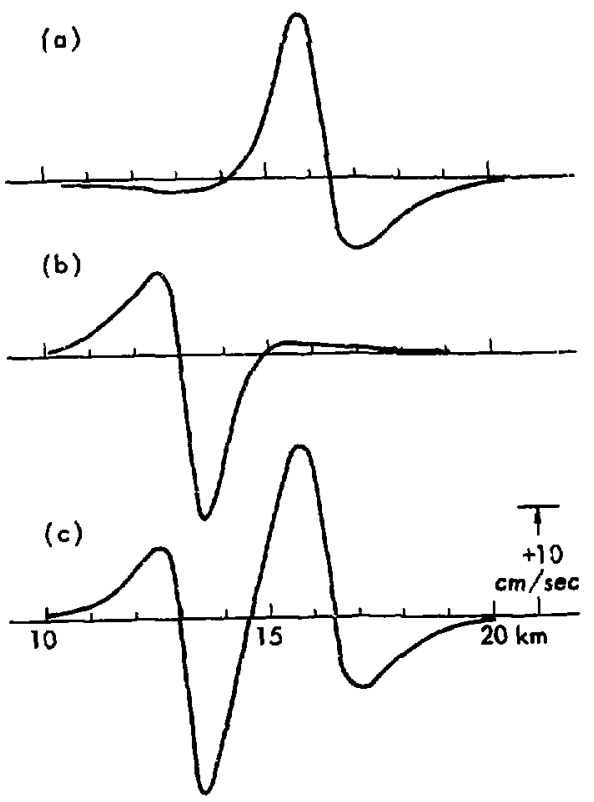

Fig. 32. Theoretical Rayleigh waves frcm (a) buried point source, (b) Eurface force or impact. and (c) superposition of both sources with surface force delayed by $2.5 \mathrm{sec}$. The vertical component of particle velocity is plotted as a function of distance from the source at time $15 \mathrm{sec}$.

the one observed in the inelastic spall cun.

\section{CONCLUSIONS}

The possibility that the impact of spalled earth ard rock is the source of the surface waves radiated by underground nuclear explosions has been examined. The mass and mumentum of the spall material has been estimated from accelerometer records. Then, using the estimateò spall momentum as the input 
impulse, range-peak displacement curves have been calculated. These calculations are based on an extension of Lamb's (1904) solution for the Rayleigh wave resulting from a normal impulse. The calculated peak displacement amplitudes are roughly the same as the experimentally observed amplitudes. This result indicates that the spall material has sufficient momentum to produce surface waves of the observed amplitude.

A kind of controlled geophysical experiment to determine the effect of spall was then performed. This was done with the aid of a large general-purpose computer program that calculates the dynamic elastic-inelastic response of earth materials. The computer calculation gave the response of the earth to an underground nuclear explosion for given input material properties. Two runs were made with the same viscosity, elastic parameters, explosion energy release, and depth of burial. The only difference between the runs was that in one case the strength of the material was limited. In the run with no tensile strength and low shear strength (at lov: pressures), spallation could and did occur. In the run without limits on the stress, spall was prevented and the material behavior was elastic. The spallation run was observed to produce Rayleigh wave amplitudes roughly 2.7 times larger than the elastic run produced, and the waves were observed to be delayed with respect to the waves in the elastic run. This experiment demonstrates that inelastic spallation processes are important in generating surface waves, even though the explosion is completely contained.

Though these computational studies and the experimental data on spall give much evidence to suppose that spall plays a strong role in the surface-wave generation process, the theory would be even more strongly supported if it could be demonstrated that there are detectable effects at teleseismic range. Parhaps the most likely effect to look for would be an anomalous time delay in the surfacewave arrivals from explosions. Recently a delay has been observed which might be explained by the spall theory. In studying surface wave arrivals from the Amchitka Island Test Site !vents, von Seggern (1973) discovered that the arrival of the Rayleigh wave from the Milrow explosion was delayed slightly with respect to the arrival of the Rayleigh wave from the Long Shot explosion. Since the travel paths are identical, it is difficult to explain the observed difference of $1.6 \pm$ $0.9 \mathrm{sec}$ except in terms of some source effect. Examination of Perret's accelerometer data from both events shows that spall occurred in both cases, and that the ballistic period for the M:Irow spall was much longer than for Long Shot. On the basis of these data, the duration of the ballistic period for Milrow is estimated to exceed that of Long Shot by $0.6 \pm$ 0.3 sec. Also, it seems likely that differences in shot depth would cause a slight additional delay. Long Shot was buried at $2300 \mathrm{ft}$ and Milrow at $\$ 000 \mathrm{ft}$. Thus the Milrow spall would also be delayed by the time it would take the blast wave to iravel the additional $1700 \mathrm{ft}$ to the surface. With a compressional velocity of $3.0 \mathrm{~km} / \mathrm{sec}$ computed from the shock arrival time on the accelerometer record, the depth delay would add about $0.2 \mathrm{sec}$, to give a total delay of 0.8 $\pm 0.3 \mathrm{sec}$. Thus there is a fair amount 
of overlap between the range of delays observed in the teleseismic signals and the range of delays estimated from accelerometer records on the basis of the spall theory of generation. More experi- mental delay studies of this type are ne:essary to tell whether or not teleseismic surface-wave delays between pairs of explosions correlate well with accelerometer data on spall impact delays. 


\section{Site Geometry Effects}

\section{A. INTRODUCTION}

In addition to understanding the basic processes by which the Rayleigh wave is generated, one would also like to investigate some of the effects man might be able to produce. It might, for example, be possible to alter the generating process by making changes in the spatial configuration of the test site. Since much time and effort had already been expended in aralyzing and verifying the computational methods for Rayleigh wave calculations it was decided to use this tool in an inyestigation of site geometry. There are several ways the geometry could be altered. A single explosive could be replaced by vertical or horizontal arrays of multigle explosions. Similarly, the surface curvature could be controlled to some extent by choice of site locations.

With the intention of keeping the study as brief and systematic as possible, it was decided to consider only three explosion configurations in combiration with three types of surface curvature. The surface curvature was realized by highly idealized models of mountain and canyon or crater topograpnies. Altogether, nine runs were made. The Rayleigh-wave amplitude for each case was then compared with the amplitude calculated from an initial reference run. The reference run was chosen to correspond to the normal site configuration: a single explosion detonated bencath relatively flat ground. Fach run had the same total explosive ent:rgy release. Thus one could estimate the Rayleigh-wave generating efficlency as a function of site geometry under the constraint of fixed total energy release.

\section{B. CALCULATIONS}

The calculations were confined to viscoelastic behavior. In the beginning of this study, it had been hoped it would be possible to include failure effects and spall to allow a more realistic modeling of the surface-wave generating mechanism. A spall model must, however, include gravitation, or the ballistic spall would not fall back to the surface. Gravitation, in iurn, requires the inclusion of a lithostatic stress distribution in the initial conditions, in order to maintain equilibrium. When the surface profile is irregular, corresponding to mountains or canyons, the determination of the equilibrium lithostatic stress is a difficult problem in itself, especially when the material strength is low. The inclusion of lithostatic stress and gravitation, in the case of an irregular surface, would require a two-part calculation. The initial step wculd be the iterative solution for the equilibrium stress field beneath the mountain or canyon. The result of this calculation would then be used as the initial condition for the explosion run. Although it is easy to alter the dynamic program to an iterative scheme for calculating steady-state stress distributions, the scheme that can easily be produced is equivalent to simultaneous relaxation, which is not very efficient. It was concluded that, to get the accuracy required within a reasonable time, it would have 
been necessary to go to a more efficient method such as successive overrelaxation. Because of this, it was decided to run calculations without gravitational forces and to bypass the failure routine in the program, thus confining the material behavior to viscoelastic response.

The remaining problems with numerical technique were the same as those faced in Chapter $\mathrm{V}$ and were solved in the same way. In this study it was again necessary to model the explosion neighborhood reasonably well and to be able to calculate the surface wave out to a distance of 15 to $20 \mathrm{~km}$. So that the Rayleigh wave would become well-defined and wellseparated from other waves. The solution was to use the same basic 200-m mesh discussed in Chapter $\mathrm{V}$ and illustrated in Fig. 30 (with some distortion in the source region to produce the necessary surface curvature for the mountain and canyon runs). The accuracy of the results obtained on this mesh was checked again by making one run with the difference intervals halved and comparing it with results obtained on the $200-\mathrm{m}$ mesh. The comparison test again showed differences of no more than $\pm 5 \%$ in the disp?acements, which is sufficiently acckrate for the purpose of this study.

The modeling of the explosion(s) must, at some level, be an approximation. With respect to seismology, the most important parameters of the explosion(s) are the total energy release, the depth of burst, and the site location. Keeping in mind the limitations of the numerical method, it was decided to formulate the aim of the study in a slightly less restrictive way. The aim would be, given a certain amount of total energy release and the constraint that this energy release must occur below the minimum depth required for containment of an actual explosion, to find out how the amplitude of the Rayleigh wave is influenced by the distribution of this energy in and around various topographical features.

Except for ths depth constraint, the shape and volume of the region of energy release are taken to be arbitrarily variable, since one might expect that many small explosions could be used in place of one large explosion to approximate the same energy release over an extended region. One might also expect that the energy release over an extended region could be controlled in time, however, in this study, the energy release is assumed to take place simultaneously throughout the source region. Given this formulation of the problem, it was decided to model the explosion(s) with an initial compression put into a number of zones surrounding the detonation point(s). The compression was such that the strain energy of the compressed material equaled $20 \mathrm{kt}$. The total energy of compression is approximately given by

$$
\mathbf{E}=(1 / 2) k \mathrm{~V}_{\mathrm{o}} \theta^{2}
$$

whers $\mathrm{k}$ is the bulk modulus, $V_{\mathrm{o}}$ is the volume of the uncompressed material and $\theta$ is the dilatation. Table 4 gives the source volume, energy density, and compression for each run. The enerky density given in the table is the energy per unit volume, referred to the volums the material would occupy if it were in the uncompressed state; the volume given is the actual volume, $V$, of the compressed 
material. The two are related by

$$
v_{0}=V(1+|\theta|) .
$$

In all cases, the energy density of the compressed material is very low because the volume of material compressed is very large. The energy densities range between 0.162 and $1.23 \mathrm{~atm}$, and volumes range between 0.678 and $5.146 \mathrm{~km}^{3}$. The vertical and horizontal source volumes are nearly identical and are approximately 7 times bigger than the concentrated source volumes, Outside the compressed region, the energy density is initially zero, because there is no lithostatic stress distribution.

Figure 33 indiicates the configuration of each source. The mesh lines in the source neighbortood are plotted, and those zones containing initially compressed material are shaded. The calculations assume that the left edge of the mesh is an axis of cylindrical symmetry perpendicular to the surface of the Earth. The complete mesh of $22 \times 80$ zones extends to a depth of $15,0 \mathrm{~km}$ and a range of
$30.0 \mathrm{~km}$, but only the source region is shown in the figure. Each case is drawn to the same scale. The mountain is $2.0 \mathrm{~km}$ high and $4.0 \mathrm{~km}$ in diameter, and the canyon or crater is $2.0 \mathrm{~km}$ deep and $4.0 \mathrm{~km}$ in diameter. These models are highly idealized, but this does rot matter much for a study of comparative effecis. The vertically extended source in Fig. 33 approximates the simultaneous detonation of a set of individual shots spaced at equal intervals along the same drill hole. The horizontally extended source in Fig. 33 approximates the simultaneous detonation of a disk-shaped horizontal array of shots. The small-square source approximates a single explosion. These extended sirain-energy distributions represent a kind of smoothed-out equivalent source for calculating phenomena not occurring within the elastic radius of the actual explosions. For convenient labeling. these three types of sources are referred to as line, disk, and point sources.

The volume of the compressed matter in the concentrated source is roughly the

Table 4. Basic parameters for each run.

\begin{tabular}{llll}
\hline \multicolumn{1}{c}{ Source } & $\begin{array}{c}\text { Vol, } \\
\mathrm{km}^{3}\end{array}$ & $\begin{array}{c}E_{V_{\circ}} \\
\mathrm{atm}\end{array}$ & $|\theta|=|\Delta \rho| / \rho_{0}$ \\
\hline Point in plain & 0.678584 & 1.227435 & 0.0061021 \\
Point in mountain & 0.678584 & 1.227435 & 0.0061021 \\
Point in canyon & 0.678584 & 1.227435 & 0.0061021 \\
Line in plain & 5.061106 & 0.165207 & 0.0022344 \\
Line in mountain & 5.145928 & 0.162437 & 0.0022159 \\
Line in canyon & 4.538031 & 0.184227 & 0.0023596 \\
Disk in plain & 1.660562 & 0.175625 & 0.0023038 \\
Disk in mountain & 4.760562 & 0.175625 & 0.0023038 \\
Disk in canyon & 4.760552 & 0.175625 & 0.0023038 \\
\hline
\end{tabular}


same as the volume of the elastic sphere for a megaton explosion and the total elastic energy of $20 \mathrm{kt}$ is roughly equal to
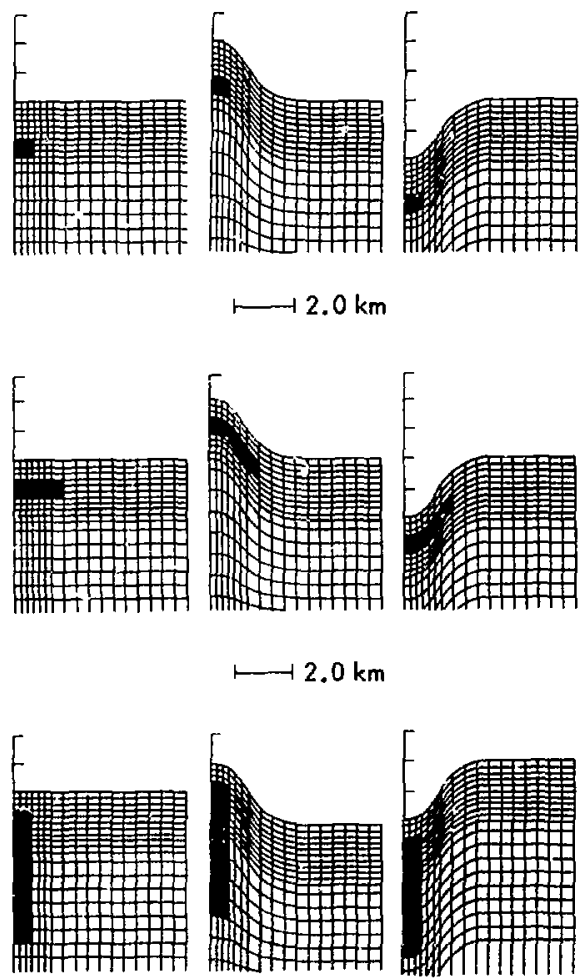

Fig. 33. Topography and source geometry for each of the nine runs. Each drawing shows a vertical cross section through the model Earth. The left edge of each section is an axis of cylindrical symmetry. The topographical features were created by distorting the Lagrangian computing mesh. Plain, mountain, and crater models are represented in the left, middle, and right columns, respectively. A concentrated source, a horizontally distributed source, and a vertically distributed source are represented in the top, niddle, and bottom rows, respectively. the sum of the stored and radiated elastic energy for a megaton explosion in tuff. These estimates are consistent with results obtained from the formulas and tables for the stored and radiated elastic energy given in Haskell (1967). The point source is centered at a depth of $1500 \mathrm{~m}$, which corresponds to a scaled depth of burial equal to $150 \mathrm{~m} / \mathrm{kt}^{1 / 3}$. This value is consistent with tests conducted in the past (Springer and Kinnaman, 1971). The horizontally and vertically distributed sources represent arrays of individual explosions of smaller yield, such that the total energy release rcmains fixed at $20 \mathrm{kt}$, and so are placed closer to the surface. The energy density of the line and disk sources is roughly one-seventh that of the point source. Therefore, the minimum depth of these sources is scaled from the point source by multiplying by the inverae cube root of 7. Representative values of density, Poisson's ratio, and bulk modulus for tuff are $1.9 \mathrm{~g} / \mathrm{cm}^{3}, 0.33$, and $66.33 \mathrm{kbar}$, respectively. These values were used in sach run. The magnitudes of the bulk and shear viscosities were increaged somewhat from the values used in Chapter $V$ to $1.37 \times 10^{9}$ and $9.12 \times 10^{8}$ poise, respectively.

\section{RESULTS AND CONC LUSIONS}

The Rayleigh-wave generating efficien: cies of the different sources were compar. $d$ by plotting the spatial variation of the vertical surface displacement at a time of 16.0 sec. By this time the Rayleigh way: is far from the source, is well separated trom the compression wave, and is approaching an asymptotic form. 
Dispersion is negligible because the model Earth has uniform material properties. Figures 34,35 , and 36 show the vertical component of the surface displacement $r$ lotted against range for the point, vertical line, and horizontal disk sources, respectively. Each figure shows three wave forms: one for the flat plain, one for the mountain, and one for the canyon. The bar chart in Fig. 37 compares the maximum peak-to-peak displacement for each case, normalized to the maximum peak-to-peak displacement produced by the point source in a flat plain. The bars corresponding to the same topography are grouped together.

Comparison of the Rayleigh-wave forms in Figs. 34, 35, and 36 shows that, except for the canyon runs, the general wave shapes and periods are not much different. The canyon runs are characterized by a leading double-negative phase. This double-negative phase

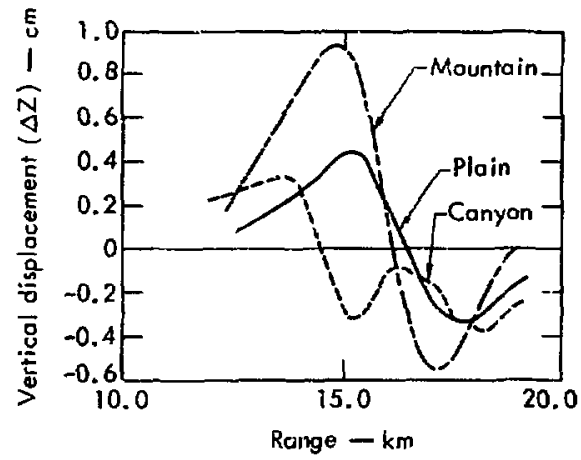

Fig. 34. The Rayleigh signals from a concentrated source of dilatation. 'The calculated spatial variation of the verticaldisplacement wave forms are plotted at 16.0 sec for the plain, mountain, and canyon runs.

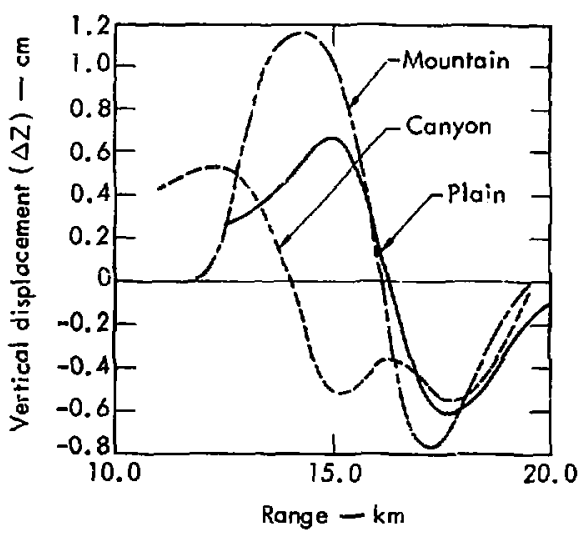

Fig. 35. The Rayleigh aignals from a vertically-distributed source of dilatation. The calculated spatial variation of the verticaldisplacement waveforms are plotted at $16.0 \mathrm{sec}$ for the plain, mountain, and canyon runs.

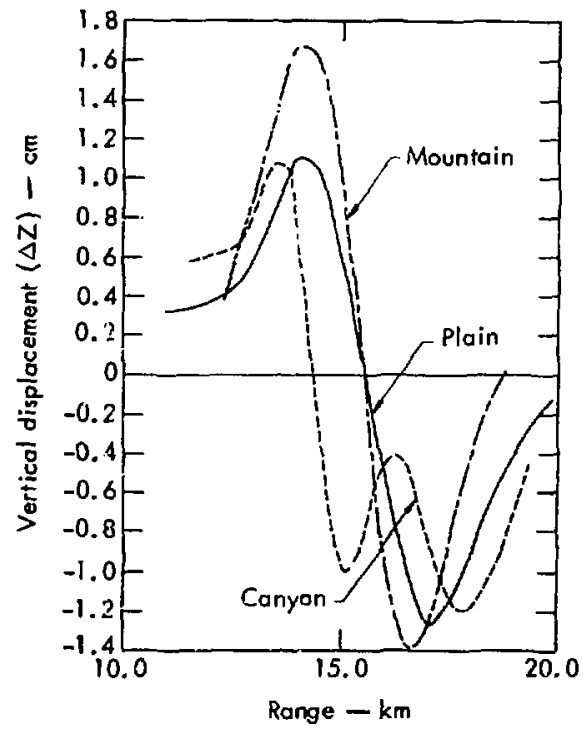

Fig. 36. The Rayleigh signals from a horizontally-distributed source of dilatation. The calculated spatial variation of the vertical displacement waveforms are plotted at $16.0 \mathrm{sec}$ for the plain. mountain, and canyon runs. 


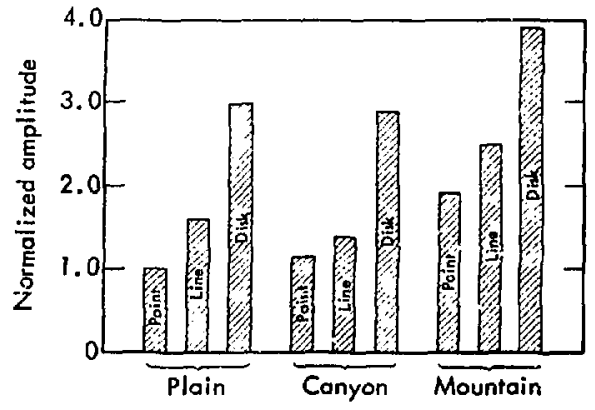

Fig. 37. Comparison of the peak-to-peak Rayleigh vertical-displacement amplitudes for each of the nine runs at $16.0 \mathrm{sec}$. The amplitudes are normalized by diviring by the amplitude obtainis from the concentrated source beneath a flat plain. The energy release at the source is the same in each case. The vertically-distributed sources (bars labeled "Line") and the horizontally-distributed sources (bars labeled "Disk") each have seven times the volume of the concentrated sources (bars labeled "Point"). The runs corresponding to the same topography are grouped together.

occurs irrespective of the configuration of the source-energy distribution. A leading negative phase, corresponding to displacement downward into the Earth, occurs in all the runs. This is consistent with the results obtained jy Cagniard (1962) for the asymptotis Rayleigh wave produred by a buried point suurce of dilatation.

The emplitudes of the wayes are affected by the volumie ard orientation of the explosion source and the topography. Figure 37 shows that there is nearly a factor of 4 difference in amplitude between the strongest and weakest signals obtained. The figure shows that the amplitude increases with increased volume and that the source orientation strongly controls the rate of increase. In the case of the flat plain, increasing the volume of the concentrated source sevenfold by expanding it downward produced a $60 \%$ increase in amplitude, whereas distributing the same volume-increase horizontally produced a $200 \%$ increase in amplitude. Similar results obtain for the canyon and mountain models. Figure 37 also shows that the fopography alone can afrect the amplitude. Although the canyon amplitudes are not much different from the flat-plain amplitudes, the mountain amplitudes are iarger. In particular, firing the point source beneath the mountain results in a $90 \%$ increase in amplitude. However, the effect is not proportionately as great for the horizontally-distributed source.

It may be argued that the much larger a.nplitude observed for the horizontally extended source reflects the fact that the average depth is least for this configuration. This nay be: however, a rerun of the disk-in-plain problem, with the disk at exactly the same depth as the concentrated sourc -, still produces a twofold increase in amp:itude. Whatever the under?ying causes, the study has shiw that, with thse given constraints, a combination of multiple explosion configuration and topography cail significantly alter the peak Rayleigh-wave amplitude. 


\section{Summary of Results and Conclusions}

The dimensions of spall from large nuclear explosions in Nevada tuffs, as determined from analysis of accelerometer records, were found to scale as the one-third power of the yield. The spall impulse, the parameter of partic ular interest in Rayleigh-wave generation, was found to be related to th: yield by

$$
\mathrm{I} \simeq 4.6 \times 10^{14} \mathrm{~W} \text { dyne sec, }
$$

where $I$ is the spall impulse and $W$ is the explcsion yield in kilotons.

The spall impulse derived from finitedifference calculations of the Gasbuggy and Boxsar Events was found to agree within a factor of 2 with the impulse derived from accelerometer measurements. The numerical results are quite sensitive to material properties, not all of which were experimentaliy measured, so that some discrepancy would be expected. The comparisons are satisfactory in that they indicate that no gross errors are likely in the above estimate of spall impulse.

The Rayleigh-wave amplitudes calculated from a modification of Lambs (1904) analytical solution for an impulse applied at the surface, using the above relationship for the impulse, were found to agree within a factor of 2 or less with measured Rayleigh-wave amplitudes at ranges from 30 to $600 \mathrm{kr}$. Considering the scatter in seismic data taken from different stations, this is good agreement. The computer experiments, in which Rayleigh waves were calculated with and without spall, showed that the Rayleigh waves from the spall case had much larger amplitudes and were delayed with respect to the Rayleigh waves from the elastic case. These delays appear to be consistent with the anomalous time delays in Rayleigh-wave arrivals from different nuclear explosions recently reported by von Seggern (1973, On the basis of these results, there is reason to believe that spall impulse is a significant source of Rayleigh waves from buried nuclear explosions.

The numerical study of topograpiny and explosion-ccinfiguration effects showed considerable differences in the amplitudes of Rayleigh waves generated for the same energy release. In particular, the disk-like array fired under a mountain was found to produce four times the surface-wave amplitude obtained from a single explosion under flat ground.

With respect to numerical work, the analysis of the linear $-Q$ artificial viscosity showed that it strongly affects the shape of decaying blast waves and surface waves. The traditional artificial viscosity uses coefficien:s that are functions of mesh size, so that attempts to calculate weak decaying waves will produce different answers on different meshes. To calculate weak decaying waves accurately, it is necessary to replace the linear $-Q$ artificial viscosity with a model that is independent of purely numerical parameters and that is representative of a true physical damping mechanism. The Voigt Kelvin model is quite useful for spall calculations, because the momentum carried by the blast wave can be shown to be independent of the magnitude of the viscosity 
coefficients. Thus, spall impulse can be calculated, although the magnitude of the viscosity coefficient may not be accurately known. 


\section{Acknowledgments}

I am especially grateful to John Killeen for the help, advice, and guidance extended to me in negotiating the various acader,ic hurdles and in preparing this dissertation. Equal thanks are due Willard J. Hannen and Howard $C$. Rodean for their critical comment and review.

This worli was performed under the auspices of the United States Atomic Energy Commission at the Irawrence Livermore Laboratory. I am grateful for the latitude granted me and the confidence shown in me by my supervisors during the performance of this work. In particular, the support of Thomas
Wainwright, William C. Grayson, Howard C. Rodean, and William F. Noh is gratefully acknowledged.

Accelerometer data were kindly supplied by William R. Perret of Sandia Laboratories, Albuquerque, New Mexico. Seismic data were supplied by Environmental Resea.ch Corporation, Las Vegas, Nevada. The comments and suggestions of William Perrot and Donald L. Springer regarding the interpretation of experimental data are greatly appreciated.

I would like to thank James Cheney, Robert Fisher, and Larry 'ragan for helpful comments and editorial assistance in the writing of this dissertation. 


\section{Bibliography}

Alterman, 7.S. and J. Aboudi, Seismic pulse in a layered sphere; normal modes and surface waves, J. Geophys, Res. 74, 2618-2636 (1969).

Brune, J.N., A.E. Spinosa, and J. Oliver, Relative excitation of surface waves by earthquakes and underground explosions in the California-Nevada region, J. Geophys. Res. 68, 3501-3513 (1963).

Butkovich, T.R., The Gas Equation of State for Natural Materials, Lawrence Livermore Laboratory, Rept. UCRL-14729 (1967).

Butkovich, T.R., Influence of water in rocks on effects of underground nuclear explosions, J. Geophys. Res. 76, 1993-2011 (1971).

Cagniard, L., Reflection and Refraction of Progressive Seismic Waves, E. A. Flinn and C.H. Dix, transl. (McGraw-Hill, New York, 1962), pp, 171-182.

Carpenter, E.W., R.A. Savill, and J. K. Wright, The dependence of seismic signal amplitudes on the size of underground explosions, Geophys. J. Roy. Astron. Soc. $6,426-440$ (196?).

Cherry, J. T., S. Sack, G. Maenchen, and V. Kransky, Two-Dimensional Stres8:Induced Adiabatic Flow, Lawrence Livermore Laboratory, Rept. UCRL-50987 (197i).

Cherry, J. T., Computer calculation of explosion produced craters, Int. J. Rock Mech. Min. Sci. 4, 1-22 (1967).

Chilton, F., J.D. Eisler, and H.G. Heubacil, Dymamics of spalling of the Earths's surface caused by underground explosions, J. Geophys. Reg. 71, 5911-5919 (1966).

Douglas, A., J.A. Hudson, and V.K. Kembhavi, The relative excitation of seismic surface and body waves by point sources, Geophys. J. Roy. Astron. Soc. 23, $451-460$ (1971).

Eisler, J. D, and F. Chilton, Spalling of the Earth's surface by underground nuclear explosions, J. Geophys, Res. 69, 5285-5293 (1964).

Eisler, J. D., F. Chilton, and F. M. Saur, Multiple subsurface spalling by underground nuclear explosions, J. Geophys, Res. 71, 3923 -3927 (1966).

Evernden, J. F., Identification of earthquakes and explosions by the use of teleseismic data, J.Geophys. Res. 74, 3828-3856 (1969).

N. Ewing, S. Jardetzky, and F. Press, Elastic Waves in Layered Media (McGraw-Hill, New York, 1957), Chapter 2.

Garvin, W. W., Exact transient solution of the buried line source problem. Proc. Roy. Soc. (London) Ser. A, 234, 528-541 (1956).

Handin, J., H. C. Heard, and J. N. Magouirk, Effecrs of the intermediate principal stress on the failure of limestone, dolomite, and glass at different temperatures and strain rates, J. Geophys. Res. 72, 611-640 (1967).

Hannon, W. J., An examination of Rayleigh Waves Produced by Shear and Compressional Line Sources, Lawrence Livermore Laboratory, Rept. UCRL-51233 (1972). 
Harkrider, D. G., Surface waves in multilayered elastic media, I, Rayleigh and Love waves from buried sources in a multilaycred half-space, Bull. Sulsmol. Soc. Amer. 54, 627-679 (1964).

Haskell, N. A., Analytic approximation for the elastic radtation from a conlained underground explosion, J. Geophys. Res. 72, 2583-2587 (1967).

Heard, H. C. . R. N. Schock, and D. R. Stephens, High-Pressure Mechanical Propertics of Tuff from the Dlamond Mine Site, Lawrence Livermore Laboratory, Rept. UCRL-51090 (1971).

Johnson, G. W.. G. H. Higgins, and C. E. Violet, Underground nuclear detonations, J. Geophys. Aes. 64, 1457-1470 (1958).

Knopof,, L., Q, Rev. Geophys. 2, 625-660 (1969).

Lamb, H., On the propagation of tremors over the surlace of an elastic solid, Phil. Trans. Roy. Soc. (London) Ser. A, 203, 1-42 (1904).

Liebermann, R.C. and P.W. Pomeroy, Relative excitation of surface waves by earthquakes and underground explosions, J. Geophys. Res. 74, 1575-1580 (1969).

Lifshitz, J.M. and H. Kolsky, The propagation of spherically divergent stress pulses in linear viscoelastic solids, J. Mech. Phys, Solids 13, 361-376 (1965).

Lighthill, M. J., "Viscosity Efrects in Sound Waves of Finite Amplitude," in Surveys in Mechanics, G. K. Batchelor and R.M. Davies, Eds. (Cambridge University Press, Cambridge, Mass., 1956), pp, 250-351.

Maenchen, G. and S. Sack, "The Tensor Code," in Methods in Computational Physins, B. Alder, S. Fernback, and M. Rotenberg, Eds. (Academic Press, New York, 1964), pp. 181-210.

Marshall, P. D., Aspects of the spectral differences between earthquakes and undorground explosions, Geophys. J. Roy. Astron. Soc. 20, 397-416 (1970).

Nakano, J. H., On Rayleigh waves, Jap. J. Astr. Geophys. 2, 233 (1825).

Newlands, M., Lamb's problem with internal dissipation: I, J. Acoust. Soc. Amer. 26. $434-498$ (1954).

Perret, W. R.. Gasbugty Selsmic Source and Surface Motion, Clearinghousc for liederal Scientific and Technical Information, Rept. PNE-1002. Nationa! Bureau of Standards, U.S. Department of Commerce, Springrield, Virginia (1969).

Perrel, W. R., Free-field and Surface Molion from Nuclear Explosion in Alluyium: Merlin Event, Sandia Laboratories, Albuquerque, New Mexico, Research Rept. SC-RR-69-334 (1971), pp. 113-138.

Press, F., G. Dewart, and R. Gilman, A study of diagnostic techniques for tdontifying earthquakes, J. Geophy. Res. 66, 2909-2928 (1963).

Richtmyer, R. D. and K.W. Morton, Difference Methods for Initial-Value Problems (Interscience, New Yoitk, 1967), and ed., pp. 311-338.

Rinehart, J.S., How to predict spalling when caused by large blasts, Eng. Mining .1. 161. 8, 98-101 (1960). 
Hodean, H. C., Nurlear-Explosion Sucismology, ABC Critical Review Series (U.S. Atomic lincray Commission, Techniral informalion Center, Oak Nidge, Tennessee, 19711, pp. 9-43.

sherwoud, J.W. C., Hastic wave propagation in a semi-infinite solid medium, Proc. Pliys. Sac. Londos 71, 207-219 (1950).

Stluil, Seismic Mellods for Moniluring linderground Explosions, D. Diviles, Ed. (International institute lor Peare and Conflirl Kesearch, Stockholm, 1968).

Springer, D. L. and K. L. Kinnaman, Seismic snurce summary for the U.S. Underground surlear explosions, Aull. Seismal. Soc. Am. 61, 1073-1098 (1971).

Stupizens, D. R.. II. Louts, and E. M. L.illey. Loading-Unlonding PV Curves for Tuffs from the Nevada Test Sile, Iawrence Livermore Laboralory. Repl. UCRL-SOSSA (1969a).

Stephens, T. R., H. Louis, and H. M. Lilley, Pressure-Volume Relationships for Tuffe and Phyoliteg from the Nevada Test Site, Lawrence Livermore Laboratory. Rept. UCAL-5057日 (1969b).

Stephens, D. R., H. C. Heard, and R. N. Schock, Migh-Pressure Mechanical Properties of furr from the Diamond Dust Site, Lawrence Livermore Laboratory. Rept. UCRL,-5085á (1970a).

Stephens, D. R., H. M. Lilley, and H. Louis, Pressure-volume equation of state of consolidated and fractured rocks to $40 \mathrm{~kb}$, Int. J. Rock Mech. Min. Sci. I $257-296(1970 b)$.

Tanyi, G. E.. Rayleigh waves generated by an explosion in an elastic sp!tere, Gsophys. J. Roy. Astron. Soc. 10, 485-49S (1966).

Terhune, R. W., T. F. Stubbs, and J,T. Cherry. Nuciear Cratering on a Digital Computer. Lawrence Livermore laboratory. Rept, UCRL-S0898 (1970).

Thiruvenkatachar, V.R. and $K$. Viswanathan, Dynamic response of an elastic half space to tima dependent surface tractions over an embedded spherical cavity, III. Proc. Noy. Soc. London) Ser. A. 309, 313-329 (1960).

Toksioz, M. N., Ben-Menahem, and D. G. Ifarkrider, Determination of source parameters of explosions and earthquakes by amplitude equalization of selsmic surface waves, I, underground nuclear explosions, J. Geophys. Res. 69, 4355-4366 (1964).

Tsai, Y. M. and H. Kolsky, Surface wave propagation for linear viscoelastic solids, 1. Mech. Phys. Solids 16, 99-109 (1968).

Vicrelli, I. A., Application of the Tensor Code to the Calculation of Rayleigh Waves, f.awrence Livermore Laboratory, Rept. UCRL-50992 (1971).

Viccelli, 1.A., Spallation and the generation of surface waves by an inderground explosion, J. Geophys. Res. 78, 2475-2487 (1973a).

Viccelli, J.A., The linear $Q$ and the calculation of decaying spherical shock in solids, J. Comp. Phys. 12, 187-201 (1973b).

Vlecelli, I. A. Topography and the Rayleigh-wave generating efficiency of buriedexplosive sources, J. Geophys. Res. 8, 3334-3339 (1973c). 
Von Seggern, D., Scismic surface waves from Amchitka Island Test Site Bvents and their relation to source mechanism. J. Geophys. Mes. 78, 2467-2474 (1973).

Werth, G.C., R. F. Herbst, and D. L. Springer, Amplitudes of seismie arrivals from the M discontinuity, J. Geophys, Res. 67, 15137-1610 (1962).

Werth, G.C. and R. F. Herbst, Comparison of amplitudes of seismic waves from nucleir explosions in four mediums, I. Geophys. Aes. 6E, 1463-1475 (1963).

Whitn, J. W., An invarient description of failure for an isotropic medium. 1. Geophys. Res. 78, 2438. 2441 (1973).

Whilkins, M. i.. "Calculation of Elastic-Plastic Flow," in Methods in Computational Physies, 13. Alder, S. Fernback, and M. Rotenberg. Fds. (Academic Press, New York, 1064), Vol, 3, pp. 211-263.

Willins, M., Salculation of Elastic-Plastic Flow; Sawrence Livermore Laboratory, Rept. UC.RL-7322, Rev. 1 (1969).

Zel'dovich, Y.B. and Y,P. Raizer, Physics of Shock Waves and High Temperature Hydrodynamic Phenomena (Academic Press, Inc., New York, 1966-1967), Vols, 1 and 2. 